\title{
THIRD ORDER OPEN MAPPING THEOREMS AND APPLICATIONS TO THE END-POINT MAP
}

\author{
FRANCESCO BOAROTTO, ROBERTO MONTI, AND FRANCESCO PALMURELLA
}

\begin{abstract}
This paper is devoted to a third order study of the end-point map in subRiemannian geometry. We first prove third order open mapping results for maps from a Banach space into a finite dimensional manifold. In a second step, we compute the third order term in the Taylor expansion of the end-point map and we specialize the abstract theory to the study of length-minimality of sub-Riemannian strictly singular curves. We conclude with the third order analysis of a specific strictly singular extremal that is not length-minimizing.
\end{abstract}

\section{INTRODUCTION}

The most challenging open problems in sub-Riemmanian geometry, such as Sard's problem and the regularity of length-minimizing curves, are related to our limited understanding of the end-point map, see $[26,1]$. In this work, we extend the analysis of the end-point map from the second to the third order. In a preliminary part of independent interest, we study open mapping theorems of the third order for maps from a Banach space into a manifold.

Let $M$ be a smooth manifold and $\Delta \subset T M$ be a totally non-holonomic (i.e., completely non-integrable) distribution with rank $2 \leq k<\operatorname{dim}(M)$. For every point $q_{0} \in M$, there exist a neighborhood $U \subset M$ of $q_{0}$ and linearly independent smooth vector fields $f_{1}, \ldots, f_{k} \in \operatorname{Vec}(U)$ such that $\Delta=\operatorname{span}\left\{f_{1}, \ldots, f_{k}\right\}$ on $U$. The distribution $\Delta$ is non-holonomic (i.e., it satisfies the Hörmander condition) if

$$
\operatorname{Lie}_{q}\left\{f_{1}, \ldots, f_{k}\right\}=T_{q} M \text { for every } q \in U,
$$

where $\operatorname{Lie}_{q}\left\{f_{1}, \ldots, f_{k}\right\}$ denotes the evaluation at $q$ of the Lie algebra generated by $f_{1}, \ldots, f_{k}$. Given $q \in U$, we say that $\Delta$ has step $s \in \mathbb{N}$ at $q$ if, to recover the equality in (1.1), we need Lie brackets of length $s$ and $s$ is the least integer with this property. We say that $\Delta$ has step $s$ on $U$ if $\Delta$ has step less than or equal to $s$ at every $q \in U$.

We fix on $\Delta$ the metric that makes $f_{1}, \ldots, f_{k}$ orthonormal. A curve $\gamma \in A C([0,1] ; U)$ is admissible if $\dot{\gamma} \in \Delta_{\gamma}$ a.e. on $[0,1]$. In this case, we have

$$
\dot{\gamma}=\sum_{i=1}^{k} u_{i} f_{i}(\gamma), \quad \text { a.e. on }[0,1]
$$

for some unique vector of functions $u \in L^{1}\left([0,1] ; \mathbb{R}^{k}\right)$, called the control of $\gamma$. The length of $\gamma$ is length $(\gamma):=\|u\|_{L^{1}\left([0,1] ; \mathbb{R}^{k}\right)}$. Since our considerations are local around a reference curve $\gamma$, in the sequel we will assume $U=M$.

Date: July 26, 2019.

2010 Mathematics Subject Classification. 46A30, 53C17, 49K15.

Key words and phrases. Open mapping theorems, end-point map, sub-Riemannian geometry.

The first author has been supported by University of Padova STARS Project "Sub-Riemannian Geometry and Geometric Measure Theory Issues: Old and New". 
Fix a point $q_{0} \in M$ and let $X=L^{1}\left([0,1] ; \mathbb{R}^{k}\right)$. The end-point map is the map $F=F_{q_{0}}$ : $X \rightarrow M$ defined by $F(u)=\gamma(1)$ where $\gamma$ is the unique solution of $(1.2)$ such that $\gamma(0)=q_{0}$. The curve $\gamma$ is said to be singular (or abnormal) if the corresponding control $u$ is a critical point of the differential $d_{u} F: X \rightarrow T_{F(u)} M$, i.e., if the differential is not surjective. The corank of $u$ is the dimension of $T_{F(u)} M / \operatorname{Im}\left(d_{u} F\right)$. Singular curves do not depend on the metric fixed on $\Delta$ but nontheless they may be length-minimizers. They do not have a counterpart in Riemannian geometry and do not obey the classical Hamiltonian formalism.

The Sard's problem investigates the size (dimension, measure, structure) of the set of points of $M$ that are reachable from $q_{0}$ by singular curves. Even though Sard's theorem does not hold in infinite-dimensional spaces [19], it is expected that for the end-point map this set is not too big, see $[22,10,32]$.

Another important problem is the regularity of length-minimizing curves. Montgomery first showed in [25] the existence of smooth strictly singular curves that are in fact length-minimizing. For the notion of strict singularity we defer to Definition 4.1. For these curves, however, the first order necessary conditions provided by the Pontryagin Maximum Principle [31] do not typically give any further regularity beyond the starting one (Lipschitz or AC). Some results on the regularity of singular sub-Riemannian geodesics are in $[23,28,18,30,9]$, see also the surveys $[29,27]$. The difficulty of the problem, again, lies in the complicated structure of the end-point map at critical points.

Similar problems are addressed e.g. in $[14,15]$, where the authors study generic properties of singular trajectories, and in [13,8,12], where some regularity results are established for the more general class of control systems affine in the control. A different approach towards singular length-minimizing curves can be found in $[2,11,4]$, where the authors follow the topological viewpoint rather than the differential one, and study singular curves via homotopy theory and results à la Morse. In the case of Carnot groups, singular curves are contained in the zero set of specific polynomials, see [20,21].

The second order analysis of the end-point map was developed by Agrachev and Sarychev in [6]. This theory provides necessary conditions for strictly singular length-minimizers. These conditions are deduced from second order open mapping theorems that exploit the notion of regular zero together with Morse's index theory [5, Chapter 20]. This is the starting point of our work.

In a first step, in Section 2, we prove abstract third order open mapping theorems for functions $F: X \rightarrow M$, where $X$ is a Banach space and $M$ a smooth manifold. In Definition 2.4, we introduce an intrinsic notion of third differential $\mathcal{D}_{0}^{3} F: \operatorname{dom}\left(\mathcal{D}_{0}^{3} F\right) \rightarrow \operatorname{coker}\left(d_{0} F\right)$, where $\operatorname{dom}\left(\mathcal{D}_{0}^{3} F\right) \subset \operatorname{ker}\left(d_{0} F\right)$ is a precise subspace of the kernel of the differential of $F$ at $0 \in X$. Then, we adapt the notion of regular zero to the third differential. For a given isotropic vector of the second differential $w_{0} \in \operatorname{Iso}\left(\mathcal{D}_{0}^{2} F\right)$, in Definition 2.7 we introduce the notion of $w_{0}$-regular zero.

Theorem 1.1. Let $X$ be a Banach space and $U \subset X$ an open neighborhood of the origin. Let $F: U \rightarrow M$ be a smooth mapping having a critical point at 0 of corank $h \geq 1$. Then:

(i) If $h=1$ and there exists $v \in \operatorname{dom}\left(\mathcal{D}_{0}^{3} F\right)$ such that $\mathcal{D}_{0}^{3} F(v) \neq 0$, then $F$ is open at the origin.

(ii) For any $h \geq 1$, if there exist $w_{0} \in \operatorname{Iso}\left(\mathcal{D}_{0}^{2} F\right)$ and $v_{0} \in \operatorname{dom}\left(\mathcal{D}_{0}^{3} F\right)$ such that $v_{0}$ is a $w_{0}$-regular zero for $\mathcal{D}_{0}^{3} F$, then $F$ is open at the origin.

The first statement is proved in Section 2.2, while the latter is shown in Section 2.3. Notice that the two statements are different in nature: indeed the first one does not use the notion of regular zero. Also, point (ii) can be seen as a more geometric version of the third order open mapping theorem proved by Sussmann in [35]. Its rephrasement in algebraic terms can 
be found in Theorem 2.8. However, this algebraic version is less satisfactory than its second order counterpart, where the notion of index of a quadratic form produces conditions that can be applied effectively to the end-point map. In our case, finding sufficient conditions of the algebraic type ensuring the existence of a regular zero for a vector valued cubic map (polynomials of degree 3) seems a difficult task.

In Section 3, we use tools of chronological calculus to compute the third order term in the Taylor expansion of the end-point map, see Proposition 3.5. In fact, our procedure is algorithmic and can be used, in principle, to compute also higher order terms. We shall see that, differently from the second order, the representation of the third differential in terms of Lie brackets is not unique. However, the scalarizations onto the cokernel of the first differential are uniquely defined.

Theorem 1.1 and the formula for the third differential of the end-point map yield the following necessary condition satisfied by any adjoint curve of a singular length-minimizing trajectory $\gamma$ of corank 1. The construction of adjoint curves is recalled in Section 4 . We denote by $d_{u} F$ the differential of the end-point map $F: L^{1}\left([0,1] ; \mathbb{R}^{k}\right) \rightarrow M$ starting from $\gamma(0)=q_{0}$, and computed at the point $u \in L^{1}\left([0,1] ; \mathbb{R}^{k}\right)$, the control of $\gamma$.

Theorem 1.2. Let $(M, \Delta, g)$ be a sub-Riemaniann manifold with $\Delta=\operatorname{span}\left\{f_{1}, \ldots, f_{k}\right\}$ for $f_{1}, \ldots, f_{k} \in \operatorname{Vec}(M)$. Assume that:

(i) $\gamma:[0,1] \rightarrow M$ is a strictly singular length-minimizing curve of corank 1 ;

(ii) the domain $\operatorname{dom}\left(\mathcal{D}_{u}^{3} F\right)$ is of finite codimension in $\operatorname{ker}\left(d_{u} F\right)$.

Then any adjoint curve $\lambda:[0,1] \rightarrow T^{*} M$ satisfies, for every $t \in[0,1]$ and for every $i, j, \ell=$ $1, \ldots, k$,

$$
\left\langle\lambda(t),\left[f_{i},\left[f_{j}, f_{\ell}\right]\right](\gamma(t))\right\rangle+\left\langle\lambda(t),\left[f_{\ell},\left[f_{j}, f_{i}\right]\right](\gamma(t))\right\rangle=0 .
$$

This result is proved in Section 4. Notice the nontrivial assumption (ii) on the dimension of the domain of the third differential. Condition (1.3) is the extension to the third order of the first and second order necessary conditions for length-minimality. In fact, if $\gamma$ is a corank-one singular length-minimizing curve with adjoint curve $\lambda$, then by the Pontryagin Maximum Principle we have $\left\langle\lambda, f_{j}\right\rangle=0$ identically along the curve, for every $j=1, \ldots, k$. If in addition $\gamma$ is strictly singular, then $\left\langle\lambda,\left[f_{i}, f_{j}\right]\right\rangle=0$ identically along $\gamma$, for every $i, j=1, \ldots, k$. This is known as Goh condition, see [17].

In Section 5 we show an application of the general theory to a specific example of singular curves. We recall the notion of extremal curves: a horizontal curve $\gamma$ is extremal if it has an adjoint curve $\lambda$ that satisfies the Pontryagin Maximum Principle. A length-minimizing curve is an extremal, but the viceversa needs not hold. The notion of (strict) singularity applies to extremal curves as well, see Definition 4.1.

Theorem 1.3. Consider on $M=\mathbb{R}^{3}$ the distribution $\Delta=\operatorname{span}\left\{f_{1}, f_{2}\right\}$, where

$$
f_{1}=\frac{\partial}{\partial x_{1}} \quad \text { and } \quad f_{2}=\left(1-x_{1}\right) \frac{\partial}{\partial x_{2}}+x_{1}^{p} \frac{\partial}{\partial x_{3}},
$$

and $p \in \mathbb{N}$. Fix on $\Delta$ the metric $g$ that makes $f_{1}$ and $f_{2}$ orthonormal. Then:

(i) For any $p \geq 2$ the curve $t \mapsto \gamma(t)=(0, t, 0)$ is a strictly singular extremal in $\left(\mathbb{R}^{3}, \Delta\right)$.

(ii) If $p$ is an even integer then $\gamma$ is locally length-minimizing in $\left(\mathbb{R}^{3}, \Delta, g\right)$.

(iii) If $p=3$ then $\gamma$ is not locally length-minimizing in $\left(\mathbb{R}^{3}, \Delta, g\right)$.

Using Theorem 1.1, or alternatively Theorem 1.2, we show that when $p=3$ the end-point map is open at the control of $\gamma$. For $p=5,7, \ldots$, the curve $\gamma$ is probably not length-minimizing. To prove this we would need open mapping theorems of order higher than 3 . 


\section{THIRD ORDER OPEN MAPPING THEOREMS}

2.1. Intrinsic third differential. Let $(X,\|\cdot\|)$ be a Banach space and let $U \subset X$ be an open neighborhood of the origin. We consider a smooth mapping $F: U \rightarrow M$, where $M$ is a smooth manifold of dimension $m \in \mathbb{N}$. Here and hereafter, by "smooth" we always mean " $C^{\infty}$-smooth".

By fixing a local chart for $M$ centered at $F(0)$, we may consider the representative of $F$ in this chart as a map from $U$ to $\mathbb{R}^{m}$, and accordingly consider its $k$-th directional derivatives $d_{0}^{k} F: X \rightarrow \mathbb{R}^{m}$

$$
d_{0}^{k} F(v):=\left.\frac{d^{k}}{d t^{k}} F(t v)\right|_{t=0}, \quad v \in X .
$$

We denote by $\left(v_{1}, \ldots, v_{k}\right) \mapsto d_{0}^{k} F\left(v_{1}, \ldots, v_{k}\right)$ the associated $k$-multilinear maps. Then we may expand $F$ as a Taylor series at 0 :

$$
F(v)=\sum_{j=0}^{k} \frac{1}{j !} d_{0}^{j} F(v)+o\left(\|v\|^{k}\right) .
$$

For $k \geq 2$, the maps $d_{0}^{k} F$ do not behave tensorially and depend on the specific choice of the local chart of $M$.

In [5, Chapter 20], the authors study a chart-independent (or "intrinsic") notion of Hessian, by quotienting out the action of the differential. Recall that 0 is a critical point of $F$ if the differential $d_{0} F: X \rightarrow T_{F(0)} M$ is not surjective. The cokernel of $d_{0} F$ is the quotient space

$$
\operatorname{coker}\left(d_{0} F\right)=T_{F(0)} M / \operatorname{Im}\left(d_{0} F\right),
$$

and the corank of this critical point is its dimension: $\operatorname{dim}\left(T_{F(0)} M / \operatorname{Im}\left(d_{0} F\right)\right)=\operatorname{dim}(M)-$ $\operatorname{dim}\left(\operatorname{Im}\left(d_{0} F\right)\right)$. The central definition for the theory is the following.

Definition 2.1. The intrinsic Hessian of $F$ at $u=0$ is the quadratic map $\mathcal{D}_{0}^{2} F: \operatorname{ker}\left(d_{0} F\right) \rightarrow$ $\operatorname{coker}\left(d_{0} F\right)$ defined by

$$
\mathcal{D}_{0}^{2} F(v):=\pi_{\operatorname{coker}\left(d_{0} F\right)}\left(d_{0}^{2} F(v)\right),
$$

where $d_{0}^{2} F$ is computed with respect to any chart centered at $F(0)$ and $\pi_{\text {coker }\left(d_{0} F\right)}$ is the projection onto coker $\left(d_{0} F\right)$.

This definition is independent of the chosen chart and for any linear form

$$
\lambda \in \operatorname{Im}\left(d_{0} F\right)^{\perp}=\left\{\lambda \in T_{F(0)}^{*} M \mid \lambda\left(d_{0} F(x)\right)=0 \text { for all } x \in X\right\},
$$

and any vector $v \in \operatorname{ker}\left(d_{0} F\right)$ there holds

$$
\lambda \mathcal{D}_{0}^{2} F(v)=\left.\mathcal{L}_{V} \circ \mathcal{L}_{V}(a \circ F)\right|_{0},
$$

where $a \in C^{\infty}(M)$ is any function such that $d_{0} a=\lambda, V \in \operatorname{Vec}(U)$ is any smooth vector field such that $V(0)=v$, and $\mathcal{L}_{V}$ denotes the Lie derivative along $V$.

We denote by $(v, w) \mapsto \mathcal{D}_{0}^{2} F(v, w)$ the bilinear form associated with the quadratic map $\mathcal{D}_{0}^{2} F(v)$.

Definition 2.2. A regular zero for the intrinsic Hessian $\mathcal{D}_{0}^{2} F$ is an element $v \in \operatorname{ker}\left(d_{0} F\right)$ such that:

(i) $\mathcal{D}_{0}^{2} F(v)=0$;

(ii) the linear map $w \mapsto \mathcal{D}_{0}^{2} F(v, w)$ is surjective from $\operatorname{ker}\left(d_{0} F\right)$ onto coker $\left(d_{0} F\right)$.

With these notions, the following theorem holds, see [5, Theorem 20.3].

Theorem 2.3 (Agrachev-Sarychev). If the intrinsic Hessian $\mathcal{D}_{0}^{2} F$ has a regular zero then $F$ is open at the origin. 
Necessary conditions for the existence of a regular zero can be found in $[7,6]$. Sufficient conditions are given by the Morse-index theory, see [5]. The existence of a regular zero is only a necessary condition for the openness of a quadratic form. For example, the map $Q: \mathbb{R}^{2} \rightarrow \mathbb{R}^{2}$ defined by $Q\left(x_{1}, x_{2}\right)=\left(x_{1}^{2}-x_{2}^{2}, 2 x_{1} x_{2}\right)$ does not have nontrivial zeros and, in particular, it has no regular zeros, but nevertheless it is open.

Our objective is to carry this program over to third-order derivatives and to deduce thirdorder sufficient conditions for the map $F$ to be open at the origin. We first need to define an "intrinsic" third differential. Let $P: M \rightarrow M$ be any diffeomorphism leaving the point $F(0)$ fixed and let $\phi:(-\varepsilon, \varepsilon) \rightarrow U$ be a smooth curve such that $\phi(0)=0$. Let us fix a local chart for $M$ centered at $F(0)$. Here and hereafter, we assume that $F(0)=0$. Then, locally in this chart, we have

$$
\begin{aligned}
\left.\frac{d^{3}}{d \varepsilon^{3}} P(F(\phi(\varepsilon)))\right|_{\varepsilon=0}= & d_{0} P\left(d_{0}^{3} F(\dot{\phi})+3 d_{0}^{2} F(\ddot{\phi}, \dot{\phi})+d_{0} F(\dddot{\phi})\right) \\
& +3 d_{0}^{2} P\left(d_{0}^{2} F(\dot{\phi})+d_{0} F(\ddot{\phi}), d_{0} F(\dot{\phi})\right) \\
& +d_{0}^{3} P\left(d_{0} F(\dot{\phi}), d_{0} F(\dot{\phi}), d_{0} F(\dot{\phi})\right) .
\end{aligned}
$$

The third derivative in the left hand-side of (2.2) transforms on $T_{0} M$ as a tangent vector (i.e., according to the first differential $d_{0} P$ only) as soon as $\dot{\phi} \in \operatorname{ker}\left(d_{0} F\right)$. Moreover, a good definition of the third differential should only depend tensorially on tangent vectors. This means that the third derivative

$$
\left.\frac{d^{3}}{d \varepsilon^{3}} F(\phi(\varepsilon))\right|_{\varepsilon=0}=d_{0}^{3} F(\dot{\phi})+3 d_{0}^{2} F(\ddot{\phi}, \dot{\phi})+d_{0} F(\dddot{\phi})
$$

should only depend on $\dot{\phi}$. This happens when $d_{0}^{2} F(\ddot{\phi}, \dot{\phi})=0$ modulo the image of $d_{0} F$. These considerations motivate the following definition.

Definition 2.4 (Intrinsic third differential). Let $F: U \rightarrow M$ be a smooth map. The domain of the third differential of $F$ at $u=0$ is:

$$
\operatorname{dom}\left(\mathcal{D}_{0}^{3} F\right):=\left\{v \in \operatorname{ker}\left(d_{0} F\right) \mid \pi_{\operatorname{coker}\left(d_{0} F\right)}\left(d_{0}^{2} F(v, x)\right)=0 \text { for all } x \in X\right\} .
$$

where $d_{0}^{2} F$ is computed with respect to any chart centered at $F(0)$. The third differential of $F$ at $u=0$ is the cubic map $\mathcal{D}_{0}^{3} F: \operatorname{dom}\left(\mathcal{D}_{0}^{3} F\right) \rightarrow \operatorname{coker}\left(d_{0} F\right)$ defined by

$$
\mathcal{D}_{0}^{3} F(v):=\pi_{\operatorname{coker}\left(d_{0} F\right)}\left(d_{0}^{3} F(v)\right),
$$

where $d_{0}^{3} F$ is computed with respect to any chart centered at $F(0)$ and $\pi_{\operatorname{coker}\left(d_{0} F\right)}$ is the projection onto coker $\left(d_{0} F\right)$.

Remark 2.5. Similarly to the Hessian, these definitions do not depend on the chosen chart. In particular we stress that, while $d_{0}^{2} F$ depends on the chart, the condition $\pi_{\text {coker }\left(d_{0} F\right)}\left(d_{0}^{2} F(v, x)\right)=$ 0 for all $x \in X$ is independent of this choice.

To see this, we proceed similarly as in (2.2), and we consider smooth curves $\phi, \psi:(-\varepsilon, \varepsilon) \rightarrow U$ such that $\phi(0)=\psi(0)=0, \dot{\psi}=v \in \operatorname{ker}\left(d_{0} F\right)$ and $\dot{\phi}=x \in X$. Also, we consider $P: M \rightarrow M$ to be any diffeomorphism fixing $F(0)=0$ and we fix a local chart around 0 . Then, by polarization, it is not difficult to see that

$$
\left.\frac{d^{2}}{d \varepsilon^{2}} P\left(\frac{F(\phi(\varepsilon)+\psi(\varepsilon))-F(\phi(\varepsilon)-\psi(\varepsilon))}{4}\right)\right|_{\varepsilon=0}=d_{0} P\left(d_{0}^{2} F(v, x)-d_{0} F\left(\frac{\ddot{\psi}}{2}\right)\right),
$$

and our assertion follows. 
As for $\mathcal{D}_{0}^{2} F$, see (2.1), for every non-zero linear form $\lambda \in \operatorname{Im}\left(d_{0} F\right)^{\perp}$ and every vector $v \in$ $\operatorname{dom}\left(\mathcal{D}_{0} F\right)$, there holds

$$
\lambda \mathcal{D}_{0}^{3} F(v)=\left.\mathcal{L}_{V} \circ \mathcal{L}_{V} \circ \mathcal{L}_{V}(a \circ F)\right|_{0}
$$

where $a \in C^{\infty}(M)$ is any function such that $d_{0} a=\lambda, V \in \operatorname{Vec}(U)$ is any smooth vector field such that $V(0)=v$, and $\mathcal{L}_{V}$ denotes the Lie derivative along $V$. Indeed, since by assumption we have $d_{0} F(v)=0$, the identity

$$
\begin{aligned}
\left.\mathcal{L}_{V} \circ \mathcal{L}_{V} \circ \mathcal{L}_{V}(a \circ F)\right|_{0} & =d_{0}^{3} a\left(d_{0} F(v)\right)+3 d_{0}^{2} a\left(d_{0}^{2} F(v), d_{0} F(v)\right)+d_{0} a\left(d_{0}^{3} F(v)\right) \\
& =\lambda d_{0}^{3} F(v) .
\end{aligned}
$$

holds. In particular, $\left.\mathcal{L}_{V} \circ \mathcal{L}_{V} \circ \mathcal{L}_{V}(a \circ F)\right|_{0}$ does not depend upon higher order differentials of $a$ at zero.

2.2. Open mapping at corank-one critical points. Assume that $u=0$ is a critical point of $F$ with corank one, i.e., $\operatorname{Im}\left(d_{0} F\right)^{\perp}$ is 1-dimensional and for some non-zero linear form $\lambda$ we have $\operatorname{Im}\left(d_{0} F\right)^{\perp}=\operatorname{span}\{\lambda\}$. To prove point (i) in Theorem 1.1, we adapt an idea used in $[5$, Lemma 20.1], which consists in finding a suitable perturbation $\phi_{\varepsilon}: X \rightarrow X$ with $\phi_{\varepsilon}(0)=0$, so that $F \circ \phi_{\varepsilon}$ is open at 0 , thus implying that $F$ is itself open at 0 .

Proof of Theorem 1.1 - (i). Since 0 is a corank-one critical point, there exists an $(m-1)$ dimensional subspace $E \subset X$, with $m=\operatorname{dim}(M)$, such that $X=E \oplus \operatorname{ker}\left(d_{0} F\right)$. Since $E$ is isomorphic to $\operatorname{Im}\left(d_{0} F\right)$ via $d_{0} F$, we identify it with $\mathbb{R}^{m-1}$. Namely, we choose a local chart for $M$ centered at $F(0)$ and we endow $T_{F(0)} M$ with a scalar product so that we may identify $T_{F(0)} M$ with $\mathbb{R}^{m}$ and $\operatorname{Im}\left(d_{0} F\right)$ with $\mathbb{R}^{m-1}$ We then fix a basis $\left(e_{i}\right)_{1 \leq i \leq m-1}$ of $E$.

Let $v \in \operatorname{dom}\left(\mathcal{D}_{0}^{3} F\right)$ be such that $\mathcal{D}_{0}^{3} F(v) \neq 0$, and let $z_{0}, z_{1} \in E$ to be fixed later. For $\varepsilon \geq 0$, we define the map $\phi_{\varepsilon}: \mathbb{R}^{m-1} \times \mathbb{R} \rightarrow X$,

$$
\phi_{\varepsilon}(x, y):=\frac{\varepsilon^{3} y^{3}}{3 !} v+\frac{\varepsilon^{6} y^{6}}{6 !} z_{0}+\frac{\varepsilon^{9} y^{9}}{9 !} z_{1}+\frac{\varepsilon^{9}}{9 !} x
$$

where $x=\left(x_{1}, \ldots, x_{m-1}\right) \in \mathbb{R}^{m-1}$ is identified with $(x, 0) \in \mathbb{R}^{m}$. Notice that $\phi_{\varepsilon}(0)=0$ for every $\varepsilon>0$. If the composition $\Phi_{\varepsilon}:=F \circ \phi_{\varepsilon}: \mathbb{R}^{m-1} \times \mathbb{R} \rightarrow M$ is open at the origin for some $\varepsilon>0$, then $F$ is a fortiori open at the origin.

We compute the Taylor expansion at zero of $\Phi_{\varepsilon}$ with respect to the parameter $\varepsilon$. The only non-trivially zero terms in this expansion are:

$$
\begin{aligned}
& \left.\Phi_{\varepsilon}^{(3)}(x, y)\right|_{\varepsilon=0}=y^{3} d_{0} F(v), \\
& \left.\Phi_{\varepsilon}^{(6)}(x, y)\right|_{\varepsilon=0}=y^{6}\left(10 d_{0}^{2} F(v)+d_{0} F\left(z_{0}\right)\right), \\
& \left.\Phi_{\varepsilon}^{(9)}(x, y)\right|_{\varepsilon=0}=y^{9}\left(280 d_{0}^{3} F(v)+84 d_{0}^{2} F\left(v, z_{0}\right)+d_{0} F\left(z_{1}\right)\right)+d_{0} F(x) .
\end{aligned}
$$

The term in the first line is zero since $v \in \operatorname{ker}\left(d_{0} F\right)$. The term in line (2.6) is also zero as soon as we choose $z_{0} \in E$ such that $d_{0} F\left(z_{0}\right)=-10 d_{0}^{2} F(v, v)$. This $z_{0}$ does exist because $v \in \operatorname{dom}\left(\mathcal{D}_{0}^{3} F\right)$ implies that $d_{0}^{2} F(v, v) \in \operatorname{Im}\left(d_{0} F\right)$. Finally, in line (2.7) we can choose $z_{1} \in E$ such that

$$
d_{0} F\left(z_{1}\right)=-84 d_{0}^{2} F\left(v, z_{0}\right) .
$$

This $z_{1}$ does exist because, again, $v \in \operatorname{dom}\left(\mathcal{D}_{0}^{3} F\right)$ implies that $d_{0}^{2} F\left(v, z_{0}\right) \in \operatorname{Im}\left(d_{0} F\right)$.

Eventually, we see that $\left.\Phi_{\varepsilon}^{(9)}(x, y)\right|_{\varepsilon=0}=280 y^{9} d_{0}^{3} F(v)+d_{0} F(x)$, and this implies that $\Phi_{\varepsilon}(x, y)$ admits the expansion

$$
\Phi_{\varepsilon}(x, y)=\left.\frac{\varepsilon^{9}}{9 !} \Phi_{\varepsilon}^{(9)}(x, y)\right|_{\varepsilon=0}+R_{\varepsilon}(x, y)
$$


where the remainder term $R_{\varepsilon}(x, y)$ is $O\left(\varepsilon^{10}\right)$ as $\varepsilon$ tends to zero. Let us define the function $\Psi_{\varepsilon}: \mathbb{R}^{m-1} \times \mathbb{R} \rightarrow X$

$$
\Psi_{\varepsilon}(x, y)=\frac{1}{\varepsilon^{9}} \Phi_{\varepsilon}\left(x, y^{1 / 9}\right) .
$$

Since $\Psi_{\varepsilon}$ is the composition of $\Phi_{\varepsilon}$ with a homeomorphism, $\Phi_{\varepsilon}$ is open at the origin if so is $\Psi_{\varepsilon}$. After a linear change of coordinates the openness at the origin of $\Psi_{\varepsilon}(x, y)$ reduces to the openness of $\widehat{\Psi}_{\varepsilon}(x, y)=(x, y)+R_{\varepsilon}\left(x, y^{1 / 9}\right)$.

Given $r>0$, we denote by $B_{r} \subset \mathbb{R}^{m}$ the ball of radius $r$ centered at the origin. We show that there exists $\delta_{0}>0$ such that $B_{\delta / 2} \subset \widehat{\Psi}_{\varepsilon}\left(B_{\delta}\right)$ for all $\delta \in\left(0, \delta_{0}\right)$. This follows from the following claim:

$$
\lim _{(x, y) \rightarrow 0} \frac{R_{\varepsilon}(x, y)}{|x|+|y|^{9}}=0 .
$$

In fact, (2.2) implies that there exists $\delta_{0}>0$ such that for all $0<\delta<\delta_{0}$ and for all $(x, y) \in B_{\delta}$ we have

$$
\left|R_{\varepsilon}\left(x, y^{1 / 9}\right)\right| \leq \frac{1}{2}(|x|+|y|) \leq \frac{\delta}{2} .
$$

Then, given $\xi \in B_{\delta / 2}$ and letting $\chi_{\varepsilon}^{\xi}(x, y):=\xi+(x, y)-\widehat{\Psi}_{\varepsilon}(x, y)$, the triangle inequality implies that $\chi_{\varepsilon}^{\xi}$ maps $B_{\delta}$ into itself. It follows by the Brouwer's fixed point theorem that $\chi_{\varepsilon}^{\xi}$ has a fixed point in $B_{\delta}$ for every $\xi \in B_{\delta / 2}$, and the openness of $\widehat{\Psi}_{\varepsilon}$ follows.

We are left to show claim (2.2). The role of $y$ and $\varepsilon$ in (2.4) is symmetric, and so the partial derivatives $\left.\frac{\partial^{k}}{\partial y^{k}} \Phi_{\varepsilon}(x, y)\right|_{(x, y)=0}$ are computed by the chain rule as in (2.5), switching $\varepsilon$ and $y$. As a consequence, we have

$$
\begin{gathered}
\left.\frac{\partial^{k}}{\partial y^{k}} \Phi_{\varepsilon}(x, y)\right|_{(x, y)=0}=0, \quad k=1, \ldots, 8, \\
\left.\frac{\partial^{9}}{\partial y^{9}} \Phi_{\varepsilon}(x, y)\right|_{(x, y)=0}=280 \varepsilon^{9} d_{0}^{3} F(v, v, v) .
\end{gathered}
$$

Similarly, we see that

$$
\left.\frac{\partial}{\partial x_{i}} \Phi_{\varepsilon}(x, y)\right|_{(x, y)=0}=\frac{\varepsilon^{9}}{9 !} d_{0} F\left(e_{i}\right), \quad i=1, \ldots, m-1,
$$

and thus we arrive at the expansion

$$
\Phi_{\varepsilon}(x, y)=\frac{\varepsilon^{9}}{9 !} d_{0} F(x)+\frac{\varepsilon^{9}}{9 !} 280 y^{9} d_{0}^{3} F(v)+O(|x||y|)+o\left(|x|+|y|^{9}\right),
$$

where the big-O term $O(|x||y|)$ takes care of all the mixed derivatives in $x$ and $y$, up to the tenth order and it satisfies $O(|x||y|)=o\left(|x|+|y|^{9}\right)$. The theorem follows.

2.3. Open mapping at critical points of arbitrary corank. We turn to the case of critical points of corank higher than one, and to the proof of point (ii) in Theorem 1.1. We begin with adapting to the third order setting the notion of regular zero.

Definition 2.6. Let $F: U \subset X \rightarrow M$ be a smooth map. The isotropy space of $\mathcal{D}_{0}^{2} F$ is

$$
\operatorname{Iso}\left(\mathcal{D}_{0}^{2} F\right):=\left\{u \in \operatorname{ker}\left(d_{0} F\right) \mid \mathcal{D}_{0}^{2} F(u)=0\right\} .
$$

Given an isotropic vector $w_{0} \in \operatorname{Iso}\left(\mathcal{D}_{0}^{2} F\right)$, we define the second-order image of $F$ at $w_{0}$ as the subspace of $\operatorname{coker}\left(d_{0} F\right)$

$$
\operatorname{Im}\left(F, 2, w_{0}\right)=\operatorname{Im}\left(\mathcal{D}_{0}^{2} F\left(w_{0}, \cdot\right)\right) .
$$


Finally, we define the second-order cokernel of $F$ at $w_{0}$ as the quotient

$$
\operatorname{coker}\left(F, 2, w_{0}\right)=\operatorname{coker}\left(d_{0} F\right) / \operatorname{Im}\left(F, 2, w_{0}\right) .
$$

Note that we have $\operatorname{Im}\left(F, 2, w_{0}\right)=0$ if and only if $w_{0} \in \operatorname{ker}\left(\mathcal{D}_{0}^{2} F\right)=\left\{w_{0} \in \operatorname{ker}\left(d_{0} F\right) \mid\right.$ $\left.\mathcal{D}_{0}^{2} F\left(w_{0}, \cdot\right)=0\right\}$.

Definition 2.7. Let $w_{0} \in \operatorname{Iso}\left(\mathcal{D}_{0}^{2} F\right)$. A $w_{0}$-regular zero for $\mathcal{D}_{0}^{3} F$ is an element $v \in \operatorname{dom}\left(\mathcal{D}_{0}^{3} F\right)$ such that:

(i) $\mathcal{D}_{0}^{3} F(v)=0$;

(ii) The linear map $\pi_{\text {coker }\left(F, 2, w_{0}\right)}\left(\mathcal{D}_{0}^{3} F(v, v, \cdot)\right): \operatorname{dom}\left(\mathcal{D}_{0}^{3} F\right) \rightarrow \operatorname{coker}\left(F, 2, w_{0}\right)$ is surjective. Above, $\pi_{\operatorname{coker}\left(F, 2, w_{0}\right)}$ is the projection onto $\operatorname{coker}\left(F, 2, w_{0}\right)$,

Proof of Theorem 1.1 - (ii). We fix on $T_{F(0)} M$ a scalar product so that we can regard all the spaces coker $\left(d_{0} F\right), \operatorname{Im}\left(\mathcal{D}_{0}^{2} F\left(w_{0}, \cdot\right)\right)$ and $\operatorname{coker}\left(F, 2, w_{0}\right)$ as subspaces of $T_{F(0)} M$ with direct sums:

$$
\begin{aligned}
T_{F(0)} M & =\operatorname{Im}\left(d_{0} F\right) \oplus \operatorname{coker}\left(d_{0} F\right), \\
\operatorname{coker}\left(d_{0} F\right) & =\operatorname{Im}\left(\mathcal{D}_{0}^{2} F\left(w_{0}, \cdot\right)\right) \oplus \operatorname{coker}\left(F, 2, w_{0}\right) .
\end{aligned}
$$

Let $E_{1} \subset X, E_{2} \subset \operatorname{dom}\left(\mathcal{D}_{0}^{2} F\right)=\operatorname{ker}\left(d_{0} F\right)$ and $E_{3} \subset \operatorname{dom}\left(\mathcal{D}_{0}^{3} F\right)$ be linear subspaces such that the following mappings are linear isomorphisms:

$$
\begin{aligned}
& d F_{0}: E_{1} \rightarrow \operatorname{Im}\left(d_{0} F\right), \\
& \mathcal{D}_{0}^{2}\left(w_{0} \cdot\right): E_{2} \rightarrow \operatorname{Im}\left(\mathcal{D}_{0}^{2} F\left(w_{0}, \cdot\right)\right), \\
& \mathcal{D}_{0}^{3}\left(v_{0}, v_{0}, \cdot\right): E_{3} \rightarrow \operatorname{coker}\left(F, 2, w_{0}\right) .
\end{aligned}
$$

We identify $E_{1}=\mathbb{R}^{m_{1}}, E_{2}=\mathbb{R}^{m_{2}}$, and $E_{3}=\mathbb{R}^{m_{3}}$ with $m_{1}+m_{2}+m_{3}=m$ and with coordinates $r \in \mathbb{R}^{m_{1}}, s \in \mathbb{R}^{m_{2}}$ and $t \in \mathbb{R}^{m_{3}}$. We also identify $r \in \mathbb{R}^{m_{1}}$ with $r=(r, 0,0) \in \mathbb{R}^{m}$, and similarly for $s$ and $t$. We denote by $\bar{e}_{1}, \ldots, \bar{e}_{m_{2}}$ a basis for $E_{2}$, and by $e_{1}, \ldots, e_{m_{3}}$ a basis for $E_{3}$.

Let $\nu, \zeta, \xi, \mu, \eta_{i}, \xi_{i}, \mu_{i}, \zeta_{\ell}, \eta_{i j}$ and $\zeta_{i \ell}$ be points in $E_{1}$ to be fixed later. For $\varepsilon>0$ we define the $\operatorname{map} \phi_{\varepsilon}: \mathbb{R}^{m_{1}} \times \mathbb{R}^{m_{2}} \times \mathbb{R}^{m_{3}} \rightarrow X$ by:

$$
\begin{aligned}
\phi_{\varepsilon}(r, s, t) & =\frac{\varepsilon^{6}}{6 !} v_{0}+\frac{\varepsilon^{7}}{7 !} t+\frac{\varepsilon^{8}}{8 !} w_{0}+\frac{\varepsilon^{11}}{11 !} s+\frac{\varepsilon^{12}}{12 !} \nu+\frac{\varepsilon^{14}}{14 !} \xi+\frac{\varepsilon^{16}}{16 !} \mu+\frac{\varepsilon^{18}}{18 !} \zeta+\frac{\varepsilon^{19}}{19 !} r \\
& +\sum_{\ell=1}^{m_{2}} \frac{\varepsilon^{17}}{17 !} s_{\ell} \zeta_{\ell}+\sum_{i=1}^{m_{3}} t_{i}\left(\frac{\varepsilon^{13}}{13 !} \eta_{i}+\frac{\varepsilon^{15}}{15 !} \xi_{i}+\frac{\varepsilon^{19}}{19 !} \mu_{i}\right) \\
& +\frac{\varepsilon^{18}}{18 !} \sum_{\ell=1}^{m_{2}} \sum_{i=1}^{m_{3}} t_{i} s_{\ell} \zeta_{i \ell}+\frac{\varepsilon^{14}}{14 !} \sum_{i, j=1}^{m_{3}} t_{i} t_{j} \eta_{i j} .
\end{aligned}
$$

Then we consider the composition $\Phi_{\varepsilon}:=F \circ \phi_{\varepsilon}: \mathbb{R}^{m_{1}} \times \mathbb{R}^{m_{2}} \times \mathbb{R}^{m_{3}} \rightarrow M$. To prove that $F$ is open at the origin it is sufficient to show that, for small $\varepsilon>0, \Phi_{\varepsilon}$ is open at the origin.

We compute the derivatives of $\varepsilon \mapsto \Phi_{\varepsilon}$ and we evaluate them at $\varepsilon=0$. We use the short-hand notation $\Phi=\Phi_{\varepsilon}$ and $\phi=\phi_{\varepsilon}$. The first non-trivially zero derivative at $\varepsilon=0$ is the sixth one:

$$
\Phi^{(6)}=F^{\prime}\left[\phi^{(6)}\right]+O(\varepsilon),
$$

that for $\varepsilon=0$ gives $\Phi^{(6)}(0)=d_{0} F\left(v_{0}\right)=0$ because $v_{0} \in \operatorname{dom}\left(\mathcal{D}_{0}^{2} F\right) \subset \operatorname{ker}\left(d_{0} F\right)$. For $k=$ $7, \ldots, 19$ we have

$$
\Phi^{(k)}=F^{\prime}\left[\phi^{(k)}\right]+\sum_{h=1}^{[k / 2]} c_{h k} F^{\prime \prime}\left[\phi^{(h)}, \phi^{(k-h)}\right]+\sum_{\substack{1 \leq h \leq \ell \leq p \\ h+\ell+p=k}} c_{h \ell p} F^{\prime \prime \prime}\left[\phi^{(h)}, \phi^{(\ell)}, \phi^{(p)}\right]+O(\varepsilon),
$$


where $c_{h k}$ and $c_{h \ell p}$ are positive integers. For $k=7, \ldots, 11$ we have $\Phi^{(k)}=F^{\prime}\left[\phi^{(k)}\right]+O(\varepsilon)$. The only non-trivially zero cases are $k=7,8,11$, for which we have $\Phi^{(k)}(0)=0$. Indeed, for $k=7$ we have $d_{0} F(t)=0$ because $t \in E_{2} \subset \operatorname{ker}\left(d_{0} F\right)$; for $k=8$ we have $d_{0} F\left(w_{0}\right)=0$ because $w_{0} \in \operatorname{Iso}\left(\mathcal{D}_{0}^{2} F\right) \subset \operatorname{ker}\left(d_{0} F\right)$; for $k=11$ we have $d_{0} F(s)=0$ because $s \in E_{3} \subset \operatorname{ker}\left(d_{0} F\right)$.

For $k=12, \ldots, 17$ we have the following expansions:

$$
\begin{aligned}
& \Phi^{(12)}=F^{\prime}\left[\phi^{(12)}\right]+c_{66} F^{\prime \prime}\left[\phi^{(6)}, \phi^{(6)}\right]+O(\varepsilon) \\
& \Phi^{(13)}=F^{\prime}\left[\phi^{(13)}\right]+c_{67} F^{\prime \prime}\left[\phi^{(6)}, \phi^{(7)}\right]+O(\varepsilon) \\
& \Phi^{(14)}=F^{\prime}\left[\phi^{(14)}\right]+c_{68} F^{\prime \prime}\left[\phi^{(6)}, \phi^{(8)}\right]+c_{77} F^{\prime \prime}\left[\phi^{(7)}, \phi^{(7)}\right]+O(\varepsilon) . \\
& \Phi^{(15)}=F^{\prime}\left[\phi^{(15)}\right]+c_{69} F^{\prime \prime}\left[\phi^{(6)}, \phi^{(9)}\right]+c_{78} F^{\prime \prime}\left[\phi^{(7)}, \phi^{(8)}\right]+O(\varepsilon) \\
& \Phi^{(16)}=F^{\prime}\left[\phi^{(16)}\right]+c_{6,10} F^{\prime \prime}\left[\phi^{(6)}, \phi^{(10)}\right]+c_{79} F^{\prime \prime}\left[\phi^{(7)}, \phi^{(9)}\right]+c_{88} F^{\prime \prime}\left[\phi^{(8)}, \phi^{(8)}\right]+O(\varepsilon) \\
& \Phi^{(17)}=F^{\prime}\left[\phi^{(17)}\right]+c_{6,11} F^{\prime \prime}\left[\phi^{(6)}, \phi^{(11)}\right]+c_{7,10} F^{\prime \prime}\left[\phi^{(7)}, \phi^{(10)}\right]+c_{89} F^{\prime \prime}\left[\phi^{(8)}, \phi^{(9)}\right]+O(\varepsilon) .
\end{aligned}
$$

The equations $\Phi^{(k)}(0)=0$ lead to the following list of conditions:

$$
\begin{aligned}
& d_{0} F(\nu)+c_{66} d_{0}^{2} F\left(v_{0}, v_{0}\right)=0, \\
& d_{0} F\left(\eta_{i}\right)+c_{67} d_{0}^{2} F\left(v_{0}, e_{i}\right)=0, \quad i=1, \ldots, m_{3}, \\
& d_{0} F(\xi)+c_{68} d_{0}^{2} F\left(v_{0}, w_{0}\right)=0, \\
& d_{0} F\left(\eta_{i j}\right)+c_{77} d_{0}^{2} F\left(e_{i}, e_{j}\right)=0, \quad i, j=1, \ldots, m_{3}, \\
& d_{0} F\left(\xi_{i}\right)+c_{78} d_{0}^{2} F\left(e_{i}, w_{0}\right)=0, \quad i=1, \ldots, m_{3}, \\
& d_{0} F(\mu)+c_{88} d_{0}^{2} F\left(w_{0}, w_{0}\right)=0, \\
& d_{0} F\left(\zeta_{\ell}\right)+c_{6,11} d_{0} F^{2}\left(v_{0}, \bar{e}_{\ell}\right)=0, \quad \ell=1, \ldots, m_{2} .
\end{aligned}
$$

Both (2.10) and (2.11) origin from $\Phi^{(14)}(0)=0$.

Equation (2.8) has a solution $\nu \in E_{1}$ because the vector $v_{0} \in \operatorname{dom}\left(\mathcal{D}_{0}^{3} F\right)$ satisfies $\mathcal{D}_{0}^{2} F\left(v_{0}\right)=0$. Equation (2.9) has a solution $\eta_{i} \in E_{1}$ because, again, the points $d_{0}^{2} F\left(v_{0}, e_{i}\right)$ are in the image of the differential. For the same reason, there exist solutions $\xi, \eta_{i j}, \xi_{i}, \mu \in E_{1}$ of (2.10), (2.11), (2.12), and (2.13).

We study (2.14). Since $v_{0} \in \operatorname{dom}\left(\mathcal{D}_{0}^{3} F\right)$ we have $\pi_{\operatorname{coker}\left(d_{0} F\right)}\left(d_{0}^{2} F\left(v_{0}, x\right)\right)=0$ for all $x \in X$. Then $d_{0} F^{2}\left(v_{0}, \bar{e}_{\ell}\right)$ also belongs to the image of the differential and so there exists a solution $\zeta_{\ell} \in E_{1}$ to $(2.14)$.

Now we consider the cases $k=18,19$. In these cases, the third differential $F^{\prime \prime \prime}$ becomes relevant and we have the following expansions:

$$
\begin{aligned}
& \Phi^{(18)}=F^{\prime}\left[\phi^{(18)}\right]+\sum_{k=6}^{9} c_{k, 18-k} F^{\prime \prime}\left[\phi^{(k)}, \phi^{(18-k)}\right]+c_{666} F^{\prime \prime \prime}\left[\phi^{(6)}, \phi^{(6)}, \phi^{(6)}\right]+O(\varepsilon), \\
& \Phi^{(19)}=F^{\prime}\left[\phi^{(19)}\right]+\sum_{k=6}^{9} c_{k, 19-k} F^{\prime \prime}\left[\phi^{(k)}, \phi^{(19-k)}\right]+c_{667} F^{\prime \prime \prime}\left[\phi^{(6)}, \phi^{(6)}, \phi^{(7)}\right]+O(\varepsilon) .
\end{aligned}
$$

The equation $\Phi^{(18)}(0)=0$ leads to the following conditions:

$$
\begin{aligned}
& d_{0} F(\zeta)+c_{6,12} d_{0}^{2} F\left(v_{0}, \nu\right)+c_{666} d_{0}^{3} F\left(v_{0}, v_{0}, v_{0}\right)=0, \\
& d_{0} F\left(\zeta_{i \ell}\right)+c_{7,11} d_{0}^{2} F\left(e_{i}, \bar{e}_{\ell}\right)=0 .
\end{aligned}
$$

We can fix $\zeta, \zeta_{i \ell} \in E_{1}$ solving (2.15), (2.16). Here we use the fact that $\mathcal{D}_{0}^{3} F\left(v_{0}\right)=0$. 
Finally, we require that $\mu_{i} \in E_{1}$ solves the equation

$$
d_{0} F\left(\mu_{i}\right)+c_{6,13} d_{0}^{2} F\left(v_{0}, \eta_{i}\right)+c_{7,12} d_{0}^{2} F\left(e_{i}, \nu\right)=0 .
$$

In this way we have $\Phi^{(19)}(0)=d_{0} F(r)+c_{8,11} d_{0}^{2} F\left(w_{0}, s\right)+c_{667} d_{0}^{3} F\left(v_{0}, v_{0}, t\right)$, so that the map $\Phi_{\varepsilon}$ has the following expansion

$$
\Phi_{\varepsilon}(r, s, t)=\varepsilon^{19}\left(d_{0} F(r)+c_{8,11} d_{0}^{2} F\left(w_{0}, s\right)+c_{667} d_{0}^{3} F\left(v_{0}, v_{0}, t\right)\right)+O\left(\varepsilon^{20}\right),
$$

with $c_{8,11} \neq 0$ and $c_{667} \neq 0$. It follows that the map $\Psi: \mathbb{R}^{m_{1}} \times \mathbb{R}^{m_{2}} \times \mathbb{R}^{m_{3}} \times \mathbb{R} \rightarrow M$

$$
\Psi(r, s, t ; \varepsilon)=\varepsilon^{-19} \Phi_{\varepsilon}(r, s, t)
$$

is of class $C^{1}$, with $\Psi(0)=0$ and such that the Jacobian $J_{(r, s, t)} \Psi(0)$ is surjective onto $T_{0} M$.

By the implicit function theorem, there exists $\varepsilon_{0}>0$ and $C^{1}$-functions $(r, s, t):\left(-\varepsilon_{0}, \varepsilon_{0}\right) \rightarrow$ $\mathbb{R}^{m_{1}} \times \mathbb{R}^{m_{2}} \times \mathbb{R}^{m_{3}}$ such that, for every $\varepsilon \in\left(-\varepsilon_{0}, \varepsilon_{0}\right)$

(i) $\Psi(r(\varepsilon), s(\varepsilon), t(\varepsilon) ; \varepsilon)=0$, and

(ii) $J_{(r, s, t)} \Psi(r(\varepsilon), s(\varepsilon), t(\varepsilon) ; \varepsilon)$ is surjective onto $T_{0} M$.

This proves that $\Psi_{\varepsilon}$ is open at the origin for small $\varepsilon>0$, and eventually that $F$ is open at the origin.

Theorem 1.1 - (ii) reduces the open mapping property for $F$ at 0 to the existence of $w_{0} \in$ Iso $\left(\mathcal{D}_{0}^{2} F\right)$ such that the third differential

$$
\mathcal{D}_{0}^{3} F: \operatorname{dom}\left(\mathcal{D}_{0}^{3} F\right) \rightarrow \operatorname{coker}\left(F, 2, w_{0}\right)
$$

admits a $w_{0}$-regular zero, and since the manifold $M$ is finite-dimensional, it is enough to consider the case when the source space is finite-dimensional.

Let us recall some facts about cubic maps. Given a cubic map $P: \mathbb{R}^{N} \rightarrow \mathbb{R}^{n}$, for integers $n$ and $N$, we denote by $T: \mathbb{R}^{N} \times \mathbb{R}^{N} \times \mathbb{R}^{N} \rightarrow \mathbb{R}^{n}$ the trilinear map associated with $P$. Then there hold the following facts:

(i) For $v \in \mathbb{R}^{N}$, the differential $d_{v} P: \mathbb{R}^{N} \rightarrow \mathbb{R}^{n}$ is the linear mapping given by $d_{v} P(x)=$ $3 T(v, v, x)$, for $x \in \mathbb{R}^{N}$.

(ii) For $v \in \mathbb{R}^{N}$, the second differential $d_{v}^{2} P: \mathbb{R}^{N} \times \mathbb{R}^{N} \rightarrow \mathbb{R}^{n}$ is the vector-valued symmetric bilinear form given by $d_{v}^{2} P(x, y)=6 T(v, x, y)$, for $x, y \in \mathbb{R}^{N}$.

(iii) The third differential $d^{3} P: \mathbb{R}^{N} \times \mathbb{R}^{N} \times \mathbb{R}^{N} \rightarrow \mathbb{R}^{n}$ is the vector-valued symmetric trilinear form given by $d^{3} P(x, y, z)=6 T(x, y, z)$, for $x, y, z \in \mathbb{R}^{N}$.

(iv) The third differential defines the linear map $L: \mathbb{R}^{N} \rightarrow \operatorname{Sym}(\mathbb{R}, N)^{n}$ into the space of $n$-tuples of $N \times N$ symmetric matrices given by

$$
L(x)=d^{3} P(x, \cdot, \cdot)=6 T(x, \cdot, \cdot), \text { for } x \in \mathbb{R}^{N} .
$$

We clearly have the identity $L(x)=d_{x}^{2} P$ as vector-valued symmetric bilinear maps, for every $x \in \mathbb{R}^{N}$.

Theorem 2.8. Let $P: \mathbb{R}^{N} \rightarrow \mathbb{R}^{n}$ be a cubic map and assume that:

(i) $N \geq n+1$;

(ii) if $e_{1}, \ldots, e_{N}$ denotes the canonical basis of $\mathbb{R}^{N}$, for every non-zero $\lambda \in\left(\mathbb{R}^{n}\right)^{*}$ the quadratic forms $Q_{i}^{\lambda}: \mathbb{R}^{N} \rightarrow \mathbb{R}$, for $i=1, \ldots, N$,

$$
Q_{i}^{\lambda}(x)=\lambda d^{3} P\left(e_{i}, x, x\right)
$$

do not have common isotropic vectors $x \neq 0$,

then $P$ has a regular zero. 
Proof. Since $N \geq n+1$ and $P$ is a cubic map, $P$ has a non-trivial zero $v \in \mathbb{R}^{N}$ by the Bézout theorem (see, e.g., [33, Theorem 1, Chapter IV §2]). We claim that this zero is regular.

Suppose by contradiction that $v$ is not regular, i.e., there exists a non-zero $\lambda \in\left(\mathbb{R}^{n}\right)^{*}$ such that

$$
\lambda d_{v} P(x)=3 \lambda T(v, v, x)=0, \text { for } x \in \mathbb{R}^{N} .
$$

Denoting by $\langle\cdot, \cdot\rangle$ the scalar product on $\mathbb{R}^{N}$, we recall the identity (compare with (2.17))

$$
\lambda T(u, v, w)=\langle u, \lambda L(v) w\rangle, \text { for } u, v, w \in \mathbb{R}^{N} .
$$

Cycling the variables in (2.18), we deduce that $\lambda T(v, x, v)=\langle v, \lambda L(x) v\rangle=0$ for every $x \in \mathbb{R}^{N}$, i.e., $v$ is a common isotropic vector for the quadratic forms $L(x)$ as $x$ varies in $\mathbb{R}^{N}$, which contradicts (ii).

Remark 2.9. In the case of scalar cubic maps, that is $P: \mathbb{R}^{N} \rightarrow \mathbb{R}$, Theorem 2.8 can be made more precise. Indeed, if $N \geq 2$, one can prove that the following are equivalent:

(i) $P$ has a regular zero.

(ii) $P$ is not a perfect cube.

(iii) The linear map $L: \mathbb{R}^{N} \rightarrow \operatorname{Sym}(\mathbb{R}, N)$ is of rank strictly greater than one.

We go back to the case of a smooth map $F: X \rightarrow M$.

Corollary 2.10. Let $X$ be a Banach space, $U \subset X$ a neighborhood of $0 \in X, M$ a smooth manifold, and $F: U \rightarrow M$ a smooth mapping. Assume that there exists $w_{0} \in \operatorname{Iso}\left(\mathcal{D}_{0}^{2} F\right)$ such that:

(i) $\operatorname{dim}\left(\operatorname{Im}\left(F, 2, w_{0}\right)\right)+\operatorname{dim}\left(\operatorname{dom}\left(\mathcal{D}_{0}^{3} F\right)\right)>\operatorname{dim}(M)$.

(ii) For every non-zero $\lambda \in \operatorname{Im}\left(F, 2, w_{0}\right)^{\perp}$ and $v \in \operatorname{dom}\left(\mathcal{D}_{0}^{3} F\right)$ there exists $x \in \operatorname{dom}\left(\mathcal{D}_{0}^{3} F\right)$ such that $\lambda \mathcal{D}_{0}^{3} F(v, v, x) \neq 0$.

Then $F$ is open at the origin.

Proof. We assume without loss of generality that $\operatorname{dim}\left(\operatorname{Im}\left(F, 2, w_{0}\right)\right)<\operatorname{dim}(M)$ for every $w_{0} \in$ $\operatorname{Iso}\left(\mathcal{D}_{0}^{2} F\right)$. If there exists $w_{0} \in \operatorname{Iso}\left(\mathcal{D}_{0}^{2} F\right)$ such that $\operatorname{dim}\left(\operatorname{Im}\left(F, 2, w_{0}\right)\right)=\operatorname{dim}(M)$ then $F$ is open at the origin by Theorem 2.3 .

By assumptions (i) and (ii), and recalling that $\lambda \in \operatorname{coker}\left(F, 2, w_{0}\right)^{*}=\operatorname{Im}\left(F, 2, w_{0}\right)^{\perp}$, we deduce by Theorem 2.8 that for every non-zero that the mapping $d_{0}^{3} F$ has a $\operatorname{coker}\left(F, 2, w_{0}\right)$-regular zero $v \in \operatorname{dom}\left(\mathcal{D}_{0}^{3} F\right)$. Projecting onto coker $\left(d_{0} F\right)$, we deduce that $v$ is $w_{0}$-regular zero for $\mathcal{D}_{0}^{3} F$ in the sense of Definition 2.7, and the claim follows.

Remark 2.11. The conclusions of Corollary 2.10 are unsatisfactory because they are not easily exploitable in the study of the end-point map, in particular at critical points of corank higher than one.

While in the second order analysis the Morse theory provides, via the algebraic notion of index, effective sufficient conditions ensuring the open mapping property, in the third order case we lack a solid algebraic theory describing the invariants of symmetric tensors of order 3 , where not even the concepts of rank and symmetric rank necessarily coincide [34], and the diagonalization process is not clear. This makes it difficult to find effective conditions ensuring the existence of regular zeros for cubic maps.

\section{THIRD ORDER ANALYSIS OF THE END-POINT MAP}

In this section we expand the end-point map and we compute the precise structure of its third order term. The computations use the language of chronological calculus for non-autonomous vector fields, that is briefly recalled in the first subsection. 
3.1. Elements of chronological calculus. Let $M$ be a smooth manifold and let $V=\left(V_{t}\right)_{t \in[0,1]}$ be a time-dependent vector field, that is, a map $M \times[0,1] \rightarrow T M$ so that $V(q, t)=V_{t}(q) \in T_{q} M$ for every $t \in[0,1]$.

The flow of $V$ is the map $P: M \times[0,1] \times[0,1] \rightarrow M, P\left(q_{0}, t_{0}, t\right)=P_{t_{0}}^{t}\left(q_{0}\right)$, given by evaluating at time $t$ the solution to the Cauchy problem:

$$
\left\{\begin{aligned}
\dot{q}(\tau) & =V_{\tau}(q(\tau)), \\
q\left(t_{0}\right) & =q_{0} .
\end{aligned}\right.
$$

We assume for our purposes that the solution to (3.1) is defined for every $t \in[0,1]$. It is enough to assume that the vector field $V$ is smooth in the space variable and locally integrable in the time variable for problem (3.1) to have a unique solution (see, e.g., [16, Chapter 2, Theorem 1.1]).

We will adopt the point of view of operatorial calculus. In particular, we interpret points $q \in M$ as linear functionals on the algebra $C^{\infty}(M)$, that is as evaluations $q(a)=a(q)$, and we interpret diffemorphisms $B$ of $M$ as automorphisms of $C^{\infty}(M)$ defined by the formula $B a(q)=$ $a(B(q))$. Finally, we identify a vector field $V \in \operatorname{Vec}(M)$ with the derivation of the algebra $C^{\infty}(M)$ given by $a \mapsto V a$.

The Cauchy problem (3.1) can be reformulated as the following Cauchy problem of operators on $C^{\infty}(M)$ :

$$
\dot{P}_{t_{0}}^{t}=P_{t_{0}}^{t} \circ V_{t}, \quad P_{t_{0}}^{t_{0}}=\mathrm{Id},
$$

where $\circ$ is the composition of operators on $C^{\infty}(M)$ acting from left to right, i.e.:

$$
\left(q_{0} \circ \dot{P}_{t_{0}}^{t}\right) a=\left(q_{0} \circ P_{t_{0}}^{t} \circ V_{t}\right) a=V_{t} a\left(P_{t_{0}}^{t}\left(q_{0}\right)\right)
$$

for every $a \in C^{\infty}(M)$ and every $q_{0} \in M$. The characterization (3.2) of $P_{t_{0}}^{t}$ motivates the following notation:

$$
\overrightarrow{\exp } \int_{t_{0}}^{t} V_{\tau} d \tau:=P_{t_{0}}^{t}
$$

and we call $P_{t_{0}}^{t}$ the right chronological exponential of $V$. Integrating iteratively the differential equation in (3.2), we may formally expand $P_{t_{0}}^{t}$ in the following Volterra series:

$$
\begin{array}{ll}
P_{t_{0}}^{t}=\mathrm{Id}+\sum_{k=1}^{\infty} \int_{\Sigma_{k}\left(t_{0}, t\right)} V_{\tau_{k}} \circ \cdots \circ V_{\tau_{1}} d \tau_{k} \ldots d \tau_{1}, & t \geq t_{0}, \\
P_{t_{0}}^{t}=\mathrm{Id}+(-1)^{k} \sum_{k=1}^{\infty} \int_{\Xi_{k}\left(t, t_{0}\right)} V_{\tau_{k}} \circ \cdots \circ V_{\tau_{1}} d \tau_{k} \ldots d \tau_{1}, & t<t_{0} .
\end{array}
$$

where

$$
\begin{array}{ll}
\Sigma_{k}\left(t_{0}, t\right):=\left\{\left(\tau_{1}, \ldots, \tau_{k}\right) \in \mathbb{R}^{k} \mid t_{0} \leq \tau_{k} \leq \cdots \leq \tau_{1} \leq t\right\} & \text { if } t \geq t_{0}, \\
\Xi_{k}\left(t, t_{0}\right):=\left\{\left(\tau_{1}, \ldots, \tau_{k}\right) \in \mathbb{R}^{k} \mid t \leq \tau_{1} \leq \cdots \leq \tau_{k} \leq t_{0}\right\} & \text { if } t<t_{0} .
\end{array}
$$

We agree that $\Sigma(0, t)=\Sigma(t), \Xi_{k}(t, 0)=\Xi_{k}(t)$ and $\Sigma_{k}=\Sigma_{k}(1)$, that is the $k$-th dimensional simplex. The series (3.4) are to be interpreted as identities of operators on $C^{\infty}(M)$. They are never convergent unless $V_{t}=0$. However, considering only finitely many terms leads to an asymptotic expansion for the chronological exponential with a precise estimate for the remainder, see $[5, \S 2.4 .4]$. 
For fixed $t_{0}, t \in[0,1], P_{t_{0}}^{t}$ is a diffeomorphism of $M$, and we denote its inverse by $Q: M \times$ $[0,1] \times[0,1] \rightarrow M, Q\left(q, t_{0}, t\right)=Q_{t_{0}}^{t}(q)$. Differentiating in $t$ the operatorial identity $P_{t_{0}}^{t} \circ Q_{t_{0}}^{t}=\mathrm{Id}$ we obtain $\dot{Q}_{t_{0}}^{t}=-V_{t} \circ Q_{t_{0}}^{t}$, motivating the notation

$$
\overleftarrow{\exp } \int_{t_{0}}^{t}\left(-V_{\tau}\right) d \tau:=Q_{t_{0}}^{t}
$$

and we call $Q_{t_{0}}^{t}$ the left chronological exponential of $-V_{t}$. Notice that in the differential equations for $P_{t_{0}}^{t}$ and $Q_{t_{0}}^{t}$ the vector fields is composed from the right with $P_{t_{0}}^{t}$ and from the left with $Q_{t_{0}}^{t}$. Similarly as for $P$, the left-chronological exponential has the formal expansion:

$$
\begin{array}{rlrl}
Q_{t_{0}}^{t} & =\mathrm{Id}+(-1)^{k} \sum_{k=1}^{\infty} \int_{\Sigma_{k}\left(t_{0}, t\right)} V_{\tau_{1}} \circ \cdots \circ V_{\tau_{k}} d \tau_{k} \ldots d \tau_{1}, & & t \geq t_{0}, \\
Q_{t_{0}}^{t}=\mathrm{Id}+\sum_{k=1}^{\infty} \int_{\Xi_{k}\left(t, t_{0}\right)} V_{\tau_{1}} \circ \cdots \circ V_{\tau_{k}} d \tau_{k} \ldots d \tau_{1}, & t<t_{0},
\end{array}
$$

and it follows from the definitions that for any $t_{0}, t_{1} \in[0,1]$ there holds the identity:

$$
\overrightarrow{\exp } \int_{t_{0}}^{t_{1}} V_{\tau} d \tau=\overleftarrow{\exp } \int_{t_{1}}^{t_{0}}\left(-V_{\tau}\right) d \tau
$$

A tangent vector $v \in T_{q} M$ can be seen as a linear functional on the algebra $C^{\infty}(M)$, defined by the formula $v(f)=d_{q} f(v)$. Given a diffeomorphism $B$ of $M$ we denote by $B_{*}$ its differential. The tangent vector $B_{*} v \in T_{B(q)} M$ defines then an operator on $C^{\infty}(M)$ according to the formula $B_{*} v:=v \circ B$. Indeed, if $q(t)$ is a differentiable curve such that $q(0)=q$ and $\dot{q}(0)=v$, then for every $a \in C^{\infty}(M)$ we have:

$$
\left(B_{*} v\right) a=\left.\frac{d}{d t} a(B(q(t)))\right|_{t=0}=\left.\frac{d}{d t}\right|_{t=0}(q(t) \circ B) a=(v \circ B) a .
$$

Recall next that a diffeomorphism of $B: M \rightarrow M$ acts on tangent vectors and vector fields via push-forward, namely if $V \in \operatorname{Vec}(M)$ we have

$$
\left(B_{*} V\right)(B(q))=B_{*}(V(q))
$$

for every $q \in M$. We may interpret this operation in terms of operators on $C^{\infty}(M)$. The previous identity reads as the following composition of operators $q \circ B \circ B_{*} V=q \circ V \circ B$, that leads to the operatorial definition:

$$
B_{*} V:=B^{-1} \circ V \circ B
$$

For $V \in \operatorname{Vec}(M)$ and $B$ a diffeomorphism of $M$, the operator $(\operatorname{Ad} B) V$ is defined by the formula

$$
(\operatorname{Ad} B) V:=B \circ V \circ B^{-1} .
$$

In fact, $\operatorname{Ad} B^{-1}$ acts on vector fields as the push-forward of $B$, and therefore $(\operatorname{Ad} B) V$ coincides with the pull-back of $V$ by $B$.

These notions apply in particular to the maps $\operatorname{Ad} P_{t_{0}}^{t}$, allowing for the following "infinitesimal" characterization: for every $X \in \operatorname{Vec}(M)$ there holds

$$
\frac{d}{d t}\left(\operatorname{Ad} P_{t_{0}}^{t}\right) X=\left(\operatorname{Ad} P_{t_{0}}^{t}\right)\left[V_{t}, X\right]=:\left(\operatorname{Ad} P_{t_{0}}^{t}\right) \operatorname{ad}\left(V_{t}\right) X
$$

where, by definition, $\operatorname{ad}(Y) X:=[Y, X]$ denotes the (left) Lie-bracket as an operator on $\operatorname{Vec}(M)$. Thus, using the argument in $[5, \S 2.5]$, we then see that $\operatorname{Ad} P_{t_{0}}^{t}$ is the unique solution to the 
Cauchy problem on $\operatorname{Vec}(M)$

$$
\dot{A}^{t}=A^{t} \circ \operatorname{ad} V_{t}, \quad A^{t_{0}}=\mathrm{Id},
$$

and this motivates the following notation:

$$
\overrightarrow{\exp } \int_{t_{0}}^{t} \operatorname{ad} V_{\tau} d \tau:=\operatorname{Ad}\left(\overrightarrow{\exp } \int_{t_{0}}^{t} V_{\tau} d \tau\right) .
$$

3.2. Expansion of the end-point map. Let $M$ be a smooth manifold and let $f_{1}, \ldots, f_{k} \in$ $\operatorname{Vec}(M)$ be smooth vector fields on $M$. Given $u \in L^{1}\left([0,1] ; \mathbb{R}^{k}\right)$ we will use the short-hand notation $f_{u(t)}:=\sum_{i=1}^{k} u_{i}(t) f_{i}$. Note that $f_{u}$ is a time-dependent vector field as in the previous subsection.

Definition 3.1. The end-point map relative to the vector fields $f_{1}, \ldots, f_{k}$ is the map $F: M \times$ $L^{1}\left([0,1] ; \mathbb{R}^{k}\right) \rightarrow M$ given by

$$
F\left(q_{0}, u\right):=F_{q_{0}}(u):=q_{0} \circ \overrightarrow{\exp } \int_{0}^{1} f_{u(t)} d t .
$$

Recall that we are assuming that the Cauchy problem for $f_{u(t)}$ has a solution defined on the whole interval $[0,1]$. We perform a perturbation analysis of the end-point map with respect to the control variable. To this aim, recall that by the variation formula in $[5, \S 2.7,(2.28)]$ we have:

$$
F_{q_{0}}(u+v)=q_{0} \circ \overrightarrow{\exp } \int_{0}^{1}\left(f_{u(t)}+f_{v(t)}\right) d t=F_{q_{0}}(u) \circ \overrightarrow{\exp } \int_{0}^{1} \operatorname{Ad}\left(\overrightarrow{\exp } \int_{1}^{t} f_{u(\tau)} d \tau\right) f_{v(t)} d t .
$$

This motivates the following definition.

Definition 3.2. The perturbation map relative to the vector fields $f_{1}, \ldots, f_{k}$ is the map $G$ : $L^{1}\left([0,1] ; \mathbb{R}^{k}\right) \times L^{1}\left([0,1] ; \mathbb{R}^{k}\right) \times M \rightarrow M$ given by

$$
G\left(u, v, q_{1}\right):=G_{q_{1}}^{u}(v)=q_{1} \circ \overrightarrow{\exp } \int_{0}^{1} \operatorname{Ad}\left(\overrightarrow{\exp } \int_{1}^{t} f_{u(\tau)} d \tau\right) f_{v(t)} d t .
$$

The term "perturbation" is of course motivated by the fact that, by the variation formula, there holds:

$$
G\left(u, v, F_{q_{0}}(u)\right)=F_{q_{0}}(u+v),
$$

so when $q_{1}=F_{q_{0}}(u)$ and $v$ is small, $G_{F_{q_{0}}(u)}^{u}(v)$ is a small perturbation of $F_{q_{0}}(u)$. For $t \in[0,1]$, we define the time-dependent vector field

$$
g_{v(t)}^{u, t}:=\operatorname{Ad}\left(\overrightarrow{\exp } \int_{1}^{t} f_{u(\tau)} d \tau\right) f_{v(t)}=\overrightarrow{\exp }\left(\int_{1}^{t} \operatorname{ad} f_{u(\tau)} d \tau\right) f_{v(t)} .
$$

As an operator on $C^{\infty}(M), G_{q_{1}}^{u}(v)$ admits the formal expansion:

$$
G_{q_{1}}^{u}(v):=q_{1} \circ \overrightarrow{\exp } \int_{0}^{1} g_{v(t)}^{u, t} d t=q_{1} \circ\left(\mathrm{Id}+\sum_{k=1}^{\infty} \int_{\Sigma_{k}} g_{v\left(\tau_{k}\right)}^{u, \tau_{k}} \circ \cdots \circ g_{v\left(\tau_{1}\right)}^{u, \tau_{1}} d \tau_{k} \ldots d \tau_{1}\right) .
$$

Replacing $v$ by $\varepsilon v$ in (3.11) and dropping the dependence on $q_{1}$, we introduce the family of diffeomorphisms depending on the parameter $\varepsilon>0$ :

$$
G^{u}(v \varepsilon)=\operatorname{Id}+\sum_{k=1}^{\infty} \varepsilon^{k} \int_{\Sigma_{k}} g_{v\left(\tau_{k}\right)}^{u, \tau_{k}} \circ \cdots \circ g_{v\left(\tau_{1}\right)}^{u, \tau_{1}} d \tau_{k} \ldots d \tau_{1} .
$$


Now we compute a different expansion for $G^{u}(v \varepsilon)$, where the role of the Lie-brackets of $f_{1}, \ldots, f_{k}$ is more transparent. We can compute the derivative in $\varepsilon$ of $G(v \varepsilon)$ using $[5, \S 2.8$, $(2.31)]:$

$$
\frac{\partial}{\partial \varepsilon} G^{u}(v \varepsilon)=W(v ; \varepsilon) \circ G^{u}(v \varepsilon)
$$

where the vector field $W(v ; \varepsilon)$ is given by the formula

$$
W(v ; \varepsilon)=\int_{0}^{1} \operatorname{Ad}\left(\overrightarrow{\exp } \int_{0}^{t} \varepsilon g_{v(\tau)}^{u, \tau} d \tau\right) g_{v(t)}^{u, t} d t=\int_{0}^{1}\left(\overrightarrow{\exp } \int_{0}^{t} \operatorname{ad} \varepsilon g_{v(\tau)}^{u, \tau} d \tau\right) g_{v(t)}^{u, t} d t
$$

For the definition of the integral $\int_{0}^{1} V_{\tau} d \tau$ of a non-autonomous vector field $t \mapsto V_{t}$, we refer to $[5, \S 2.3]$.

Comparing (3.13) with (3.5) we deduce that:

$$
G^{u}(v \varepsilon)=\overleftarrow{\exp } \int_{0}^{\varepsilon} W(v ; \eta) d \eta=\operatorname{Id}+\sum_{n=1}^{\infty} \int_{\Sigma_{n}(\varepsilon)} W\left(v ; \eta_{1}\right) \circ \ldots \circ W\left(v ; \eta_{n}\right) d \eta_{n} \ldots d \eta_{1}
$$

Thus the formal series in (3.12) and (3.15) coincide for every $\varepsilon>0$. From formula (3.15) we deduce the following expansion for $G^{u}(v)$ as an operator on $C^{\infty}(M)$.

Lemma 3.3. For every $v \in L^{1}\left([0,1] ; \mathbb{R}^{k}\right)$ we have:

$$
G^{u}(v)=\mathrm{Id}+d_{0} G^{u}(v)+\frac{1}{2} d_{0}^{2} G^{u}(v)+\frac{1}{6} d_{0}^{3} G^{u}(v)+O\left(\|v\|_{L^{1}\left([0,1] ; \mathbb{R}^{k}\right)}^{4}\right),
$$

where

$$
\begin{aligned}
d_{0} G^{u}(v)= & \int_{0}^{1} g_{v(t)}^{u, t} d t \\
d_{0}^{2} G^{u}(v)= & \int_{\Sigma_{2}}\left[g_{v\left(\tau_{2}\right)}^{u, \tau_{2}}, g_{v\left(\tau_{1}\right)}^{u, \tau_{1}}\right] d \tau_{2} d \tau_{1}+\left(\int_{0}^{1} g_{v(t)}^{u, t} d t\right) \circ\left(\int_{0}^{1} g_{v(t)}^{u, t} d t\right), \\
d_{0}^{3} G^{u}(v)= & 2 \int_{\Sigma_{3}}\left[g_{v\left(\tau_{3}\right)}^{u, \tau_{3}},\left[g_{v\left(\tau_{2}\right)}^{u, \tau_{2}}, g_{v\left(\tau_{1}\right)}^{u, \tau_{1}}\right]\right] d \tau_{3} d \tau_{2} d \tau_{1} \\
& +2\left(\int_{\Sigma_{2}}\left[g_{v\left(\tau_{2}\right)}^{u, \tau_{2}}, g_{v\left(\tau_{1}\right)}^{u, \tau_{1}}\right] d \tau_{2} d \tau_{1}\right) \circ\left(\int_{0}^{1} g_{v(t)}^{u, t} d t\right) \\
& +\left(\int_{0}^{1} g_{v(t)}^{u, t} d t\right) \circ\left(\int_{\Sigma_{2}}\left[g_{v\left(\tau_{2}\right)}^{u, \tau_{2}}, g_{v\left(\tau_{1}\right)}^{u, \tau_{1}}\right] d \tau_{2} d \tau_{1}\right) \\
& +\left(\int_{0}^{1} g_{v(t)}^{u, t} d t\right) \circ\left(\int_{0}^{1} g_{v(t)}^{u, t} d t\right) \circ\left(\int_{0}^{1} g_{v(t)}^{u, t} d t\right) .
\end{aligned}
$$

Proof. We begin with the expansion of $W(v ; \eta)$ in $(3.14)$ as a power series in $\eta$. Thanks to [5, $\S 2.5,(2.23)]$, we obtain $W(v ; \eta)=\sum_{k=1}^{\infty} \eta^{k-1} W_{k}(v)$, where

$$
W_{1}(v)=\int_{0}^{1} g_{v(t)}^{u, t} d t \text { and } W_{k}(v)=\int_{\Sigma_{k}} \operatorname{ad} g_{v\left(\tau_{k}\right)}^{u, \tau_{k}} \circ \cdots \circ \operatorname{ad} g_{v\left(\tau_{2}\right)}^{u, \tau_{2}}\left(g_{v\left(\tau_{1}\right)}^{u, \tau_{1}}\right) d \tau_{k} \ldots d \tau_{1}, \quad k \geq 2
$$


We then compute the first three terms of the sum in (3.15):

$$
\begin{aligned}
& \int_{\Sigma_{1}(\varepsilon)} W\left(v ; \eta_{1}\right) d \eta_{1}=\sum_{h=1}^{\infty} \frac{\varepsilon^{h}}{h} W_{h}(v), \\
& \int_{\Sigma_{2}(\varepsilon)} W\left(v ; \eta_{1}\right) \circ W\left(v ; \eta_{2}\right) d \eta_{2} d \eta_{1}=\sum_{h, k=1}^{\infty} \frac{\varepsilon^{h+k}}{(h+k) k} W_{h}(v) \circ W_{k}(v), \\
& \int_{\Sigma_{3}(\varepsilon)} W\left(v ; \eta_{1}\right) \circ W\left(v ; \eta_{2}\right) \circ W\left(v ; \eta_{3}\right) d \eta_{3} d \eta_{2} d \eta_{1}=\sum_{h, k, \ell=1}^{\infty} \frac{\varepsilon^{h+k+\ell} W_{h}(v) \circ W_{k}(v) \circ W_{\ell}(v)}{(h+k+\ell)(k+\ell) \ell} .
\end{aligned}
$$

Then using these formulas in (3.15), we get

$$
\begin{aligned}
G^{u}(v \varepsilon)=\mathrm{Id} & +\varepsilon W_{1}(v)+\frac{\varepsilon^{2}}{2}\left(W_{2}(v)+W_{1}(v) \circ W_{1}(v)\right)+\frac{\varepsilon^{3}}{3}\left(W_{3}(v)+W_{2}(v) \circ W_{1}(v)\right) \\
& +\frac{\varepsilon^{3}}{6}\left(\left(W_{1}(v) \circ W_{2}(v)+W_{1}(v) \circ W_{1}(v) \circ W_{1}(v)\right)+O\left(\varepsilon^{4}\|v\|_{L^{1}\left([0,1] ; \mathbb{R}^{k}\right)}^{4}\right),\right.
\end{aligned}
$$

where the estimate on the remainder follows from Remark 3.4 below. From this formula, we can compute the directional derivatives

$$
d_{0} G^{u}(v)=\left.\frac{d}{d \varepsilon} G^{u}(v \varepsilon)\right|_{\varepsilon=0}, \quad d_{0}^{2} G^{u}(v)=\left.\frac{d^{2}}{d \varepsilon^{2}} G^{u}(v \varepsilon)\right|_{\varepsilon=0}, \quad d_{0}^{3} G^{u}(v)=\left.\frac{d^{3}}{d \varepsilon^{3}} G^{u}(v \varepsilon)\right|_{\varepsilon=0},
$$

obtaining formulas (3.17), (3.18), and (3.19).

Remark 3.4. Even if Lemma 3.3 is enough for our purposes, the computation's method in the proof is algorithmic and permits to determine the terms of any order in the expansion of $G^{u}(v \varepsilon)$. Indeed, for $k \geq 1$ we have the formal identity

$$
\int_{\Sigma_{k}(\varepsilon)} W\left(v ; \eta_{1}\right) \circ \cdots \circ W\left(v ; \eta_{k}\right) d \eta_{k} \ldots d \eta_{1}=\sum_{h_{1}, \ldots, h_{k}=1}^{\infty} \frac{\varepsilon^{h_{1}+\cdots+h_{k}} W_{h_{1}}(v) \circ \cdots \circ W_{h_{k}}(v)}{\left(h_{1}+\cdots+h_{k}\right) \ldots\left(h_{k-1}+h_{k}\right) h_{k}} .
$$

As consequence of Lemma 3.3, we obtain an explicit formula for the intrinsic third differential of $G_{q_{1}}^{u}$ (recall Definition 2.4).

Proposition 3.5. For any $v \in \operatorname{dom}\left(\mathcal{D}_{0}^{3} G_{q_{1}}^{u}\right)$ and $\lambda \in \operatorname{Im}\left(d_{0} G_{q_{1}}^{u}\right)^{\perp}$ we have:

$$
\lambda \mathcal{D}_{0}^{3} G_{q_{1}}^{u}(v)=2 \int_{\Sigma_{3}}\left\langle\lambda,\left[g_{v\left(\tau_{3}\right)}^{u, \tau_{3}},\left[g_{v\left(\tau_{2}\right)}^{u, \tau_{2}}, g_{v\left(\tau_{1}\right)}^{u, \tau_{1}}\right)\right]\left(q_{1}\right)\right\rangle d \tau_{3} d \tau_{2} d \tau_{1} .
$$

Proof. Let $v \in \operatorname{dom}\left(\mathcal{D}_{0}^{3} G_{q_{1}}^{u}\right)$ and $a \in C^{\infty}(M)$ be such that $a\left(q_{1}\right)=0$ and $d_{q_{1}} a=\lambda$. Since $\operatorname{dom}\left(\mathcal{D}_{0}^{3} G_{q_{1}}^{u}\right) \subset \operatorname{ker}\left(d_{0} G_{q_{1}}^{u}\right)$, we deduce that

$$
d_{0} G_{q_{1}}^{u}(v)=q_{1} \circ \int_{0}^{1} g_{v\left(\tau_{1}\right)}^{u, \tau_{1}} d \tau_{1}=0
$$

By the definition of the third differential and by a computation similar to (2.2) we have

$$
\left.\frac{d^{3}}{d \varepsilon^{3}} a\left(G_{q_{1}}^{u}(v \varepsilon)\right)\right|_{\varepsilon=0}=\lambda \mathcal{D}_{0}^{3} G_{q_{1}}^{u}(v) .
$$

We used (3.22) to prove that the terms involving second and third order derivatives of $a$ are zero. Moreover, as $v \in \operatorname{dom}\left(\mathcal{D}_{0}^{3} G_{q_{1}}^{u}\right)$ we also have

$$
\left.\frac{d^{2}}{d \varepsilon^{2}} a\left(G_{q_{1}}^{u}(v \varepsilon)\right)\right|_{\varepsilon=0}=\lambda \mathcal{D}_{0}^{2} G_{q_{1}}^{u}(v)=0 .
$$


Returning to the chronological notation, we have to expand to the third order the expression

$$
\left(G_{q_{1}}^{u}(v)\right) a=\left(q_{1} \circ \overrightarrow{\exp } \int_{0}^{1} g_{v(t)}^{u, t} d t\right) a .
$$

Comparing (3.16) with the expansion provided in (3.11), we have to calculate:

$$
\begin{aligned}
& 2\left(q_{1} \circ \int_{\Sigma_{2}} g_{v\left(\tau_{2}\right)}^{u, \tau_{2}} \circ g_{v\left(\tau_{1}\right)}^{u, \tau_{1}} d \tau_{2} d \tau_{1}\right) a, \text { and } \\
& 6\left(q_{1} \circ \int_{\Sigma_{3}} g_{v\left(\tau_{3}\right)}^{u, \tau_{3}} \circ g_{v\left(\tau_{2}\right)}^{u, \tau_{2}} \circ g_{v\left(\tau_{1}\right)}^{u, \tau_{1}} d \tau_{3} d \tau_{2} d \tau_{1}\right) a .
\end{aligned}
$$

From formula (3.18) in Proposition 3.3 we obtain

$$
\begin{aligned}
\left(d_{0}^{2} G_{q_{1}}^{u}(v)\right) a & =2\left(q_{1} \circ \int_{\Sigma_{2}} g_{v\left(\tau_{2}\right)}^{u, \tau_{2}} \circ g_{v\left(\tau_{1}\right)}^{u, \tau_{1}} d \tau_{2} d \tau_{1}\right) a \\
& =\int_{\Sigma_{2}}\left\langle\lambda,\left[g_{v\left(\tau_{2}\right)}^{u, \tau_{2}}, g_{v\left(\tau_{1}\right)}^{u, \tau_{1}}\right]\left(q_{1}\right)\right\rangle d \tau_{2} d \tau_{1}+d_{q_{1}}^{2} a\left(\int_{0}^{1} g_{v(t)}^{u, t}\left(q_{1}\right) d t, \int_{0}^{1} g_{v(t)}^{u, t}\left(q_{1}\right) d t\right)=0 .
\end{aligned}
$$

Indeed, since $v \in \operatorname{dom}\left(\mathcal{D}_{0}^{3} G_{q_{1}}^{u}\right)$, the second term is zero by (3.22), and moreover

$$
\frac{1}{2} \int_{\Sigma_{2}}\left[g_{v\left(\tau_{2}\right)}^{u, \tau_{2}}, g_{v\left(\tau_{1}\right)}^{u, \tau_{1}}\right]\left(q_{1}\right) d \tau_{2} d \tau_{1} \in \operatorname{Im}\left(d_{0} G_{q_{1}}^{u}\right),
$$

so that the dual product with $\lambda \in \operatorname{Im}\left(d_{0} G_{q_{1}}^{u}\right)^{\perp}$ cancels also the first one.

By (3.19), (3.22) and $\lambda \in \operatorname{Im}\left(d_{0} G_{q_{1}}^{u}\right)^{\perp}$, for the last term in (3.23) we similarly obtain the identity

$$
\begin{aligned}
\left(d_{0}^{3} G_{q_{1}}^{u}(v)\right) a & =6\left(q_{1} \circ \int_{\Sigma_{3}} g_{v\left(\tau_{3}\right)}^{u, \tau_{3}} \circ g_{v\left(\tau_{2}\right)}^{u, \tau_{2}} \circ g_{v\left(\tau_{1}\right)}^{u, \tau_{1}} d \tau_{3} d \tau_{2} d \tau_{1}\right) a \\
& =2 \int_{\Sigma_{3}}\left\langle\lambda,\left[g_{v\left(\tau_{3}\right)}^{u, \tau_{3}},\left[g_{v\left(\tau_{2}\right)}^{u, \tau_{2}}, g_{v\left(\tau_{1}\right)}^{u, \tau_{1}}\right]\right]\left(q_{1}\right)\right\rangle d \tau_{3} d \tau_{2} d \tau_{1},
\end{aligned}
$$

whence the thesis follows.

Remark 3.6. The representation formula (3.21) for $\mathcal{D}_{0}^{3} G_{q_{1}}^{u}(v)$ in terms of Lie brackets is not unique, and a different representation can be obtained in the following way. If we compute the derivative of $\varepsilon \mapsto G^{u}(v \varepsilon)$ according to $[5, \S 2.8,(2.32)]$, we find

$$
\frac{\partial}{\partial \varepsilon} G^{u}(v \varepsilon)=G^{u}(v \varepsilon) \circ \widetilde{W}(v ; \varepsilon)
$$

where

$$
\widetilde{W}(v ; \varepsilon):=\int_{0}^{1}\left(\overrightarrow{\exp } \int_{1}^{t} \operatorname{ad} \varepsilon g_{v(\tau)}^{u, \tau} d \tau\right) g_{v(t)}^{u, t} d t .
$$

Note that the composition order of $G^{u}(v ; \varepsilon)$ and $\widetilde{W}(v ; \varepsilon)$ in $(3.24)$ is reversed compared to (3.13). Since, by (3.6), we have

$$
\begin{aligned}
\overrightarrow{\exp } \int_{1}^{t} \operatorname{ad} \varepsilon g_{v(\tau)}^{u, \tau} d \tau & =\operatorname{Ad}\left(\overrightarrow{\exp } \int_{1}^{t} \varepsilon g_{v(\tau)}^{u, \tau} d \tau\right) \\
& =\operatorname{Ad}\left(-\overleftarrow{\exp } \int_{t}^{1} \varepsilon g_{v(\tau)}^{u, \tau} d \tau\right)=\overrightarrow{\exp } \int_{t}^{1}-\operatorname{ad} \varepsilon g_{v(\tau)}^{u, \tau} d \tau
\end{aligned}
$$


the expansion in Volterra series of $\widetilde{W}(v ; \varepsilon)$ is

$$
\widetilde{W}(v ; \varepsilon)=\int_{0}^{1} g_{v(t)}^{u, t} d t+\sum_{k=2}^{\infty}(-\varepsilon)^{k-1} \int_{\Sigma_{k}} \operatorname{ad} g_{v\left(\tau_{1}\right)}^{u, \tau_{1}} \circ \cdots \circ \operatorname{ad} g_{v\left(\tau_{k-1}\right)}^{u, \tau_{k-1}}\left(g_{v\left(\tau_{k}\right)}^{u, \tau_{k}}\right) d \tau_{k} \ldots d \tau_{1}
$$

In (3.25), the order of the vector fields in the commutator is reversed with respect to (3.20). Our computation also yields the identity

$$
\begin{aligned}
\int_{\Sigma_{3}}\left[g_{v\left(\tau_{3}\right)}^{u, \tau_{3}},\left[g_{v\left(\tau_{2}\right)}^{u, \tau_{2}}, g_{v\left(\tau_{1}\right)}^{u, \tau_{1}}\right]\right] d \tau_{3} d \tau_{2} d \tau_{1} & =\int_{\Sigma_{3}}\left[g_{v\left(\tau_{1}\right)}^{u, \tau_{1}},\left[g_{v\left(\tau_{2}\right)}^{u, \tau_{2}}, g_{v\left(\tau_{3}\right)}^{u, \tau_{3}}\right]\right] d \tau_{3} d \tau_{2} d \tau_{1} \\
& +\frac{1}{2}\left[\int_{0}^{1} g_{v(t)}^{u, t} d t, \int_{\Sigma_{2}}\left[g_{v\left(\tau_{2}\right)}^{u, \tau_{2}}, g_{v\left(\tau_{1}\right)}^{u, \tau_{1}}\right] d \tau_{2} d \tau_{1}\right]
\end{aligned}
$$

thanks to which we may obtain another expression for $\lambda \mathcal{D}_{0}^{3} G_{q_{1}}^{u}(v)$.

Even though the representation for the third differential is not unique, for any $v \in \operatorname{dom}\left(\mathcal{D}_{0}^{3} G_{q_{1}}^{u}\right)$ and $\lambda \in \operatorname{Im}\left(d_{0} G_{q_{1}}^{u}\right)^{\perp}$ we have the identity:

$$
\int_{\Sigma_{3}}\left\langle\lambda,\left[g_{v\left(\tau_{3}\right)}^{u, \tau_{3}},\left[g_{v\left(\tau_{2}\right)}^{u, \tau_{2}}, g_{v\left(\tau_{1}\right)}^{u, \tau_{1}}\right]\right]\left(q_{1}\right)\right\rangle d \tau_{3} d \tau_{2} d \tau_{1}=\int_{\Sigma_{3}}\left\langle\lambda,\left[g_{v\left(\tau_{1}\right)}^{u, \tau_{1}},\left[g_{v\left(\tau_{2}\right)}^{u, \tau_{2}}, g_{v\left(\tau_{3}\right)}^{u, \tau_{3}}\right]\right]\left(q_{1}\right)\right\rangle d \tau_{3} d \tau_{2} d \tau_{1}
$$

For the second differential, the two series in (3.20) and (3.25) produce the same formula, that was already established e.g. in [5, §20.3]. For further discussions concerning the algebra of all representations for the $k$ th differential we refer to [3].

\section{THIRD ORDER NECESSARY CONDITIONS FOR SINGULAR LENGTH-MINIMIZERS}

We use the Taylor formula for the end-point map obtained in Section 3, in connection with our open mapping results, to get third-order necessary conditions satisfied by strictly singular length-minimizers.

Let $f_{1}, \ldots, f_{k} \in \operatorname{Vec}(M)$ be smooth vector fields on the manifold $M$ spanning the distribution $\Delta$ and satisfying the Hörmander condition (1.1). We denote by $X=L^{1}\left([0,1] ; \mathbb{R}^{k}\right)$ the space of controls and by $J: X \rightarrow[0, \infty), J(u)=\|u\|_{L^{1}\left([0,1] ; \mathbb{R}^{k}\right)}$ the length-functional. For a fixed $q_{0} \in M$, we consider the end-point map $F=F_{q_{0}}: X \rightarrow M$. The extended end-point map is the map $\mathcal{F}: X \rightarrow M \times \mathbb{R}$ given by $\mathcal{F}(u)=(F(u), J(u))$.

Definition 4.1. A critical point $u \in X$ of $\mathcal{F}$ is regular (resp. singular) if there exists $\left(\lambda, \lambda_{0}\right) \in$ $\operatorname{Im}\left(d_{u} \mathcal{F}\right)^{\perp} \subset T_{F(u)}^{*} M \times \mathbb{R}$ such that $\lambda_{0} \neq 0$ (resp. $\left.\lambda_{0}=0\right)$. A critical point $u \in X$ is strictly singular if, for every $\left(\lambda, \lambda_{0}\right) \in \operatorname{Im}\left(d_{u} \mathcal{F}\right)^{\perp}, \lambda_{0}=0$. An extremal curve $\gamma$ is regular (resp. singular, strictly singular), if its associated control $u$ is regular (resp. singular, strictly singular).

If $u$ is strictly singular, the length-coordinate is covered by $\operatorname{Im}\left(d_{u} \mathcal{F}\right)$ and thus the intrinsic second and third differentials of the extended end-point map $\mathcal{F}$ coincide with the ones of endpoint map $F$ itself.

Let $q_{1}=F_{q_{0}}(u)$ be the final point and, as in formula (3.9), define $G_{q_{1}}^{u}: X \rightarrow M$ letting $G_{q_{1}}^{u}(v)=F_{q_{0}}(u+v)$. In this section we omit in $F$ and $G$ the subscripts $q_{0}, q_{1}$, and the superscript $u$. The openness of $\mathcal{F}$ at $u$ is thus further reduced to the openness of $G$ at 0 . By construction, we have the following identities

$$
d_{0} G=d_{u} F, \quad \mathcal{D}_{0}^{2} G=\mathcal{D}_{u}^{2} F, \quad \mathcal{D}_{0}^{3} G=\mathcal{D}_{u}^{3} F
$$


Thanks to Proposition 3.5, given $\lambda \in \operatorname{Im}\left(d_{0} G\right)^{\perp}$ the trilinear map $\lambda T: \operatorname{dom}\left(\mathcal{D}_{0}^{3} G\right)^{3} \rightarrow \mathbb{R}$ associated with $\lambda \mathcal{D}_{0}^{3} G$ is given by:

$$
\lambda T\left(v_{1}, v_{2}, v_{3}\right)=\frac{1}{3} \sum_{\substack{1 \leq i, j, k \leq 3 \\ i \neq j, j \neq k, i \neq k}} \int_{\Sigma_{3}}\left\langle\lambda,\left[g_{v_{i}\left(\tau_{3}\right)}^{u, \tau_{3}},\left[g_{v_{j}\left(\tau_{2}\right)}^{u, \tau_{2}}, g_{v_{k}\left(\tau_{1}\right)}^{u, \tau_{1}}\right]\right]\left(q_{1}\right)\right\rangle d \tau_{3} d \tau_{2} d \tau_{1} .
$$

Corollary 2.10 specializes as follows.

Proposition 4.2. Assume that there exists $w_{0} \in \operatorname{Iso}\left(\mathcal{D}_{0}^{2} G\right)$ such that:

(i) $\operatorname{dim}\left(\operatorname{dom}\left(\mathcal{D}_{0}^{3} G\right)\right)+\operatorname{dim}\left(\operatorname{Im}\left(G, 2, w_{0}\right)\right)>\operatorname{dim}(M)$;

(ii) For every non-zero $\lambda \in \operatorname{Im}\left(G, 2, w_{0}\right)^{\perp}$ and $v \in \operatorname{dom}\left(\mathcal{D}_{0}^{3} G\right)$ the real-valued map

$$
\operatorname{dom}\left(\mathcal{D}_{0}^{3} G\right) \ni x \mapsto \lambda T(v, v, x)
$$

is not the zero mapping.

Then $G$ is open at zero.

As a consequence we have the following corollary, that is of interest when $\operatorname{coker}\left(G, 2, w_{0}\right) \neq 0$ :

Corollary 4.3. Let $u$ be the control of a strictly singular length-minimizing curve. Then, for every $w_{0} \in \operatorname{Iso}\left(\mathcal{D}_{0}^{2} G\right)$ one of the following holds:

(i) $\operatorname{dim}\left(\operatorname{dom}\left(\mathcal{D}_{0}^{3} G\right)\right)+\operatorname{dim}\left(\operatorname{Im}\left(G, 2, w_{0}\right)\right) \leq \operatorname{dim}(M)$, or

(ii) there exist a non-zero covector $\lambda \in \operatorname{Im}\left(G, 2, w_{0}\right)^{\perp}$ and $v \in \operatorname{dom}\left(\mathcal{D}_{0}^{3} G\right)$ such that $\lambda T(v, v, x)=$ 0 for every $x \in \operatorname{dom}\left(\mathcal{D}_{0}^{3} G\right)$.

For strictly singular length-minimizers of corank one, the negation of Theorem 1.1 provides a more refined criterion. Indeed, its contrapositive translates into a pointwise condition as soon as the subspace $\operatorname{dom}\left(\mathcal{D}_{0}^{3} G\right)$ is sufficiently large.

Let us first recall the construction of adjoint curves. Let $\gamma:[0,1] \rightarrow M$ be an admissible curve with control $u$, with $\gamma(0)=q_{0}$ and $\gamma(1)=q_{1}$. We denote by $P_{t_{0}}^{t}$ the flow of the non-autonomous vector field $V_{\tau}=f_{u(\tau)}$ as in (3.3). Then we have $\gamma(t)=P_{0}^{t}\left(q_{0}\right)$ for $t \in[0,1]$. By our discussion in Section 3.1, we see that the differential $\left(P_{t}^{1}\right)_{*}: T_{\gamma(t)} M \rightarrow T_{q_{1}} M$ is given by

$$
\left(P_{t}^{1}\right)_{*}=\operatorname{Ad}\left(\left(P_{t}^{1}\right)^{-1}\right)=\operatorname{Ad}\left(P_{1}^{t}\right)=\operatorname{Ad}\left(\overrightarrow{\exp } \int_{1}^{t} f_{u(\tau)} d \tau\right) .
$$

The adjoint map $\left(P_{t}^{1}\right)^{*}$ sends $T_{q_{1}}^{*} M$ to $T_{\gamma(t)}^{*} M$. For every $\lambda \in \operatorname{Im}\left(d_{0} G\right)^{\perp}$, the curve of covectors defined by

$$
\lambda(t):=\left(P_{t}^{1}\right)^{*} \lambda \in T_{\gamma(t)}^{*} M, \quad t \in[0,1],
$$

is called the adjoint curve to $\gamma$ relative to $\lambda$. In the corank 1 case, this curve is unique up to normalization of $\lambda \neq 0$.

Proof of Theorem 1.2. Proving (1.3) is equivalent to show that for any $v_{1}, v_{2}, v_{3} \in \mathbb{R}^{k}$ and for $\lambda \in \operatorname{Im}\left(d_{0} G\right)^{\perp}$ we have

$$
\left\langle\lambda,\left[g_{v_{1}}^{t},\left[g_{v_{3}}^{t}, g_{v_{2}}^{t}\right]\right]\left(q_{1}\right)\right\rangle+\left\langle\lambda,\left[g_{v_{2}}^{t},\left[g_{v_{3}}^{t}, g_{v_{1}}^{t}\right]\right]\left(q_{1}\right)\right\rangle=0, \quad t \in[0,1] ;
$$

where, as in (3.10), we set $g_{v}^{t}:=\left(P_{t}^{1}\right)_{*} f_{v}$ for $t \in[0,1]$ and $v \in \mathbb{R}^{k}$. Indeed, for all $i, j, \ell=1, \ldots, k$ we have

$$
\left\langle\lambda(t),\left[f_{i},\left[f_{j}, f_{\ell}\right]\right](\gamma(t))\right\rangle=\left\langle\left(P_{t}^{1}\right)^{*} \lambda,\left[f_{i},\left[f_{j}, f_{\ell}\right]\right](\gamma(t))\right\rangle=\left\langle\lambda,\left[g_{i}^{t},\left[g_{j}^{t}, g_{\ell}^{t}\right]\right]\left(q_{1}\right)\right\rangle,
$$

where we set $g_{i}^{t}=\left(P_{t}^{1}\right)_{*} f_{i}$. 
Let us fix $\bar{t} \in[0,1)$. Given $s>0$ such that $\bar{t}+s \leq 1$, for every $v \in L^{1}\left([0,1] ; \mathbb{R}^{k}\right)$ compactly supported in $(0,1)$ we define

$$
v_{s}(t):=v\left(\frac{t-\bar{t}}{s}\right), \quad \text { for } t \in[\bar{t}, \bar{t}+s],
$$

and zero elsewhere. We consider the subspace of $\operatorname{dom}\left(\mathcal{D}_{0}^{3} G\right)$

$$
E_{s}:=\left\{u \in \operatorname{dom}\left(\mathcal{D}_{0}^{3} G\right) \mid u=v_{s} \text { for some } v \in L^{1}\left([0,1] ; \mathbb{R}^{k}\right) \text { with } \int_{0}^{1} v(t) d t=0\right\},
$$

and we observe that while $E_{s}$ depends on $s$, its codimension does not.

Given $v \in L^{1}\left([0,1] ; \mathbb{R}^{k}\right)$, its primitive $z \in A C\left([0,1] ; \mathbb{R}^{k}\right)$ with $z(0)=0$ is

$$
z(t)=\int_{0}^{t} v(\tau) d \tau, \quad t \in[0,1] .
$$

Similarly, for any $v_{s}$ as in (4.1) let $z_{s}$ be its primitive with $z_{s}(0)=0$. It is immediate to establish the identity:

$$
z_{s}(t)=s z\left(\frac{t-\bar{t}}{s}\right)
$$

Moreover, if $v_{s} \in E_{s}$ the zero-mean property of $v$ translates into:

$$
z_{s}(\bar{t})=z_{s}(\bar{t}+s)=0 .
$$

In the next lines, we shall use several times the following integration by parts formula. For every $0 \leq \alpha<\beta \leq 1$ and $v \in L^{1}\left([0,1] ; \mathbb{R}^{k}\right)$, denoting by $z \in A C\left([0,1] ; \mathbb{R}^{k}\right)$ the primitive of $v$, we have:

$$
\begin{aligned}
\int_{\alpha}^{\beta} g_{v(t)}^{t} d t & =\int_{\alpha}^{\beta} \sum_{i=1}^{k} g_{i}^{t}\left(\dot{z}_{i}(t)\right) d t \\
& =\sum_{i=1}^{k} g_{i}^{\beta} z_{i}(\beta)-\sum_{i=1}^{k} g_{i}^{\alpha} z_{i}(\alpha)-\int_{\alpha}^{\beta} \sum_{i=1}^{k}\left(\partial_{t} g_{i}^{t}\right) z_{i}(t) d t \\
& =g_{z(\beta)}^{\beta}-g_{z(\alpha)}^{\alpha}-\int_{\alpha}^{\beta}\left(\partial_{t} g^{t}\right)_{z(t)} d t .
\end{aligned}
$$

Starting from Proposition 3.5, applying this formula to $v_{s} \in E_{s}$ and using (4.4) we obtain:

$$
\begin{aligned}
\frac{1}{2} \lambda \mathcal{D}_{0}^{3} G\left(v_{s}\right)= & \iiint_{\bar{t} \leq \tau_{3} \leq \tau_{2} \leq \tau_{1} \leq \bar{t}+s}\left\langle\lambda,\left[g_{v_{s}\left(\tau_{3}\right)}^{\tau_{3}},\left[g_{v_{s}\left(\tau_{2}\right)}^{\tau_{2}}, g_{v_{s}\left(\tau_{1}\right)}^{\tau_{1}}\right]\right]\left(q_{1}\right)\right\rangle d \tau_{3} d \tau_{2} d \tau_{1} \\
= & -\iint_{\bar{t} \leq \tau_{3} \leq \tau_{2} \leq \bar{t}+s}\left\langle\lambda,\left[g_{v_{s}\left(\tau_{3}\right)}^{\tau_{3}},\left[g_{v_{s}\left(\tau_{2}\right)}^{\tau_{2}}, g_{z_{s}\left(\tau_{2}\right)}^{\tau_{2}}\right]\right]\left(q_{1}\right)\right\rangle d \tau_{3} d \tau_{2} \\
& -\iiint_{\bar{t} \leq \tau_{3} \leq \tau_{2} \leq \tau_{1} \leq \bar{t}+s}\left\langle\lambda,\left[g_{v_{s}\left(\tau_{3}\right)}^{\tau_{3}},\left[g_{v_{s}\left(\tau_{2}\right)}^{\tau_{2}},\left(\partial_{\tau_{1}} g^{\tau_{1}}\right)_{z_{s}\left(\tau_{1}\right)}\right]\right]\left(q_{1}\right)\right\rangle d \tau_{3} d \tau_{2} d \tau_{1} \\
= & \int_{\bar{t} \leq \tau_{2} \leq \bar{t}+s}\left\langle\lambda,\left[g_{z_{s}\left(\tau_{2}\right)}^{\tau_{2}},\left[g_{z_{s}\left(\tau_{2}\right)}^{\tau_{2}}, g_{v_{s}\left(\tau_{2}\right)}^{\tau_{2}}\right]\right]\left(q_{1}\right)\right\rangle d \tau_{2} \\
& +\iint_{\bar{t} \leq \tau_{3} \leq \tau_{2} \leq \bar{t}+s}\left\langle\lambda,\left[\left(\partial_{\tau_{3}} g^{\tau_{3}}\right)_{z_{s}\left(\tau_{3}\right)},\left[g_{v_{s}\left(\tau_{2}\right)}^{\tau_{2}}, g_{z_{s}\left(\tau_{2}\right)}^{\tau_{2}}\right]\right]\left(q_{1}\right)\right\rangle d \tau_{3} d \tau_{2} \\
& -\iiint_{\bar{t} \leq \tau_{3} \leq \tau_{2} \leq \tau_{1} \leq \bar{t}+s}\left\langle\lambda,\left[g_{v_{s}\left(\tau_{3}\right)}^{\tau_{3}},\left[g_{v_{s}\left(\tau_{2}\right)}^{\tau_{2}},\left(\partial_{\tau_{1}} g^{\tau_{1}}\right)_{z_{s}\left(\tau_{1}\right)}\right]\right]\left(q_{1}\right)\right\rangle d \tau_{3} d \tau_{2} d \tau_{1} \\
= & A(s)+B(s)-C(s),
\end{aligned}
$$


where $A, B$, and $C$ are defined through the last identity.

From their very definition, we see that the maps

$$
\tau \mapsto g_{i}^{\tau}=\operatorname{Ad}\left(\overrightarrow{\exp } \int_{1}^{t} f_{u(\tau)} d \tau\right) f_{i}
$$

are Lipschitz continuous for every $i=1, \ldots, k$ because their derivatives depend on time through a locally bounded vector field (compare with (3.7)). Then we have the expansion

$$
g_{i}^{\bar{t}+s \theta}=g_{i}^{\bar{t}}+O(s),
$$

where the error $O(s)$ is uniform for $0 \leq \theta \leq 1$.

Now we estimate the terms $A(s), B(s)$, and $C(s)$ appearing in (4.5). We claim that

$$
A(s)=s^{3} \int_{0}^{1}\left\langle\lambda,\left[g_{z(t)}^{\bar{t}},\left[g_{z(t)}^{\bar{t}}, g_{v(t)}^{\bar{t}}\right]\right]\left(q_{1}\right)\right\rangle d t+O\left(s^{4}\right) .
$$

To prove this identity we perform in $A(s)$ the change of variable $\tau_{2}=\bar{t}+s t$ with $t \in[0,1]$, and we use (4.3) and (4.6). With a similar argument, we show that

$$
B(s)=O\left(s^{4}\right) \quad \text { and } \quad C(s)=O\left(s^{4}\right) .
$$

We conclude that

$$
\frac{1}{2} \lambda \mathcal{D}_{0}^{3} G\left(v_{s}\right)=s^{3} \int_{0}^{1}\left\langle\lambda,\left[g_{z(t)}^{\bar{t}},\left[g_{z(t)}^{\bar{t}}, g_{v(t)}^{\bar{t}}\right]\right]\left(q_{1}\right)\right\rangle d t+O\left(s^{4}\right) .
$$

Let us introduce the set:

$$
\mathfrak{Z}:=\left\{z \in A C\left([0,1] ; \mathbb{R}^{k}\right) \mid \dot{z} \in \operatorname{dom}\left(\mathcal{D}_{0}^{3} G_{q_{1}}^{u}\right), z(0)=z(1)=0\right\} .
$$

As in (4.2), in the next lines given $z \in \mathfrak{Z}$ we set $v=\dot{z}$. By point (i) of Theorem 1.1, the map $\mathfrak{Z} \ni z \mapsto \lambda \mathcal{D}_{0}^{3} G(\dot{z})$ is the zero map. Otherwise the curve $\gamma$ would not be length-minimizing. This implies that the principal term in (4.7), i.e., the cubic map $T: \mathfrak{Z} \rightarrow \mathbb{R}$,

$$
T(z)=\int_{0}^{1}\left\langle\lambda,\left[g_{z(t)}^{\bar{t}},\left[g_{z(t)}^{\bar{t}}, g_{v(t)}^{\bar{t}}\right]\right]\left(q_{1}\right)\right\rangle d t
$$

is identically zero. By polarization, we conclude that the trilinear map $\mathfrak{T}: \mathfrak{Z} \times \mathfrak{Z} \times \mathfrak{Z} \rightarrow \mathbb{R}$ associated with $T$,

$$
\mathfrak{T}\left(z_{1}, z_{2}, z_{3}\right)=\frac{1}{6} \sum_{\substack{1 \leq i, j, k \leq 3 \\ i \neq j, j \neq k, i \neq k}} \int_{0}^{1}\left\langle\lambda,\left[g_{z_{i}(t)}^{\bar{t}},\left[g_{z_{j}(t)}^{\bar{t}}, g_{v_{k}(t)}^{\bar{t}}\right]\right]\left(q_{1}\right)\right\rangle d t,
$$

is zero as well. Integrating by parts and using the Jacobi identity, we obtain

$$
\begin{aligned}
\int_{0}^{1}\langle\lambda, & {\left.\left[g_{z_{1}(t)}^{\bar{t}},\left[g_{z_{2}(t)}^{\bar{t}}, g_{v_{3}(t)}^{\bar{t}}\right]\right]\left(q_{1}\right)\right\rangle d t=} \\
= & -\int_{0}^{1}\left\langle\lambda,\left[g_{v_{1}(t)}^{\bar{t}},\left[g_{z_{2}(t)}^{\bar{t}}, g_{z_{3}(t)}^{\bar{t}}\right]\right]\left(q_{1}\right)\right\rangle d t-\int_{0}^{1}\left\langle\lambda,\left[g_{z_{1}(t)}^{\bar{t}},\left[g_{v_{2}(t)}^{\bar{t}}, g_{z_{3}(t)}^{\bar{t}}\right]\right]\left(q_{1}\right)\right\rangle d t \\
= & \int_{0}^{1}\left\langle\lambda,\left[g_{z_{2}(t)}^{\bar{t}},\left[g_{z_{3}(t)}^{\bar{t}}, g_{v_{1}(t)}^{\bar{t}}\right]\right]\left(q_{1}\right)\right\rangle d t-\int_{0}^{1}\left\langle\lambda,\left[g_{z_{3}(t)}^{\bar{t}},\left[g_{z_{2}(t)}^{\bar{t}}, g_{v_{1}(t)}^{\bar{t}}\right]\right]\left(q_{1}\right)\right\rangle d t \\
& +\int_{0}^{1}\left\langle\lambda,\left[g_{z_{1}(t)}^{\overline{\bar{t}}},\left[g_{z_{3}(t)}^{\overline{\bar{t}}}, g_{v_{2}(t)}^{\bar{t}}\right]\right]\left(q_{1}\right)\right\rangle d t
\end{aligned}
$$


and a similar expansion holds for $\int_{0}^{1}\left\langle\lambda,\left[g_{z_{2}(t)}^{\bar{t}},\left[g_{z_{1}(t)}^{\bar{t}}, g_{v_{3}(t)}^{\bar{t}}\right]\right]\left(q_{1}\right)\right\rangle d t$, switching the role of $z_{1}$ and $z_{2}$. Plugging these expressions in (4.8), we find:

$$
2 \mathfrak{T}\left(z_{1}, z_{2}, z_{3}\right)=\int_{0}^{1}\left\langle\lambda,\left[g_{z_{1}(t)}^{\bar{t}},\left[g_{z_{3}(t)}^{\bar{t}}, g_{v_{2}(t)}^{\bar{t}}\right]\right]\left(q_{1}\right)\right\rangle+\left\langle\lambda,\left[g_{z_{2}(t)}^{\bar{t}},\left[g_{z_{3}(t)}^{\bar{t}}, g_{v_{1}(t)}^{\bar{t}}\right]\right]\left(q_{1}\right)\right\rangle d t .
$$

To conclude the proof it suffices to show that $\mathfrak{T}=0$ implies that the trilinear map $\mathfrak{A}: \mathbb{R}^{k} \times \mathbb{R}^{k} \times$ $\mathbb{R}^{k} \rightarrow \mathbb{R}$

$$
\mathfrak{A}(x, y, z)=\left\langle\lambda,\left[g_{x}^{\bar{t}},\left[g_{z}^{\bar{t}}, g_{y}^{\bar{t}}\right]\right]\left(q_{1}\right)\right\rangle+\left\langle\lambda,\left[g_{y}^{\bar{t}},\left[g_{z}^{\bar{t}}, g_{x}^{\bar{t}}\right]\right]\left(q_{1}\right)\right\rangle
$$

is zero. We now prove by contradiction that if $\mathfrak{T}=0$ then $\mathfrak{A}=0$, thus completing our argument.

Assume that there exist vectors $x, y, z \in \mathbb{R}^{k}$ such that $\mathfrak{A}(x, y, z) \neq 0$. We claim that there exists $\alpha \in C^{\infty}([0,1] ; \mathbb{R})$ such that:

(i) $\alpha x, \alpha y, \alpha z, \dot{\alpha} x, \dot{\alpha} y$ and $\dot{\alpha} z$ belong to $\mathfrak{Z}$, and

(ii) we have for some $j \neq 0$

$$
\int_{0}^{1} \alpha(t) \dot{\alpha}(t)^{2} d t=-4 \sqrt{2} j^{2} \pi^{2}
$$

To see this, let us consider the standard trigonometric basis of $L^{2}([0,1] ; \mathbb{R})$,

$$
\{1\} \cup\{\sqrt{2} \sin (2 \pi j t), \sqrt{2} \cos (2 \pi j t), \quad j=1,2, \ldots\} .
$$

Since $\operatorname{dom}\left(\mathcal{D}_{0}^{3} G\right)$ is, by assumption, of finite codimension in $\operatorname{ker}\left(d_{0} G\right)$, it is also of finite codimension in $L^{2}\left([0,1] ; \mathbb{R}^{k}\right)$, implying that for any given vector $v \in \mathbb{R}^{k}$ the set

$$
J_{v}:=\{j \in \mathbb{N} \mid \text { either } \sin (2 \pi j t) v \notin \mathfrak{Z} \text { or }(\cos (2 \pi j t)-1) v \notin \mathfrak{Z}\} \subset \mathbb{N}
$$

is finite. Our assertion follows picking $j \in\left(J_{x} \cup J_{y} \cup J_{z}\right)^{c}$ and defining $\alpha(t):=\sqrt{2}(\cos (2 \pi j t)-1)$. Finally, with $\alpha$ and $j$ chosen as above and using (4.10), we deduce from equation (4.9) that

$$
\mathfrak{T}(\alpha x, \alpha y, \dot{\alpha} z)=-2 \sqrt{2} j^{2} \pi^{2}\left(\left\langle\lambda,\left[g_{x}^{\bar{t}},\left[g_{z}^{\bar{t}}, g_{y}^{\bar{t}}\right]\right]\left(q_{1}\right)\right\rangle+\left\langle\lambda,\left[g_{y}^{\bar{t}},\left[g_{z}^{\bar{t}}, g_{x}^{\bar{t}}\right]\right]\left(q_{1}\right)\right\rangle\right) \neq 0,
$$

whence the absurd.

Remark 4.4. In accordance with the two possible expressions of $\lambda \mathcal{D}_{0}^{3} G$ given in (3.26), we observe that (1.3) is symmetric with respect to $v_{1}$ and $v_{2}$, being therefore independent of the choice of the representation.

\section{THIRD ORDER ANALYSIS OF A SINGULAR EXTREMAL}

We prove in this section Theorem 1.3. The sub-Riemannian structure $\left(\mathbb{R}^{3}, \Delta\right)$ in its statement has step $p+1$. If $p=1$ then there is no singular curve because if a covector $\lambda$ is orthogonal to $f_{1}, f_{2}$, and $\left[f_{1}, f_{2}\right]$ then it is zero, contradicting the Pontryagin Maximum Principle. If $p \geq 2$ then $\Delta$ has constant step equal to 2 away from the plane $x_{1}=0$. Then any singular extremal passes through $x_{1}=0$.

Proof of Theorem 1.3 - (i). A horizontal curve $\gamma \in A C\left([0,1] ; \mathbb{R}^{3}\right)$ satisfies, for some control $u \in L^{1}\left([0,1] ; \mathbb{R}^{2}\right)$,

$$
\dot{\gamma}_{1}=u_{1}, \quad \dot{\gamma}_{2}=u_{2}\left(1-\gamma_{1}\right), \quad \dot{\gamma}_{3}=u_{2} \gamma_{1}^{p} \quad \text { a.e. on }[0,1] .
$$

We assume that $\gamma(0)=0$ and that $|u(t)|=1$ for a.e. $t \in[0,1]$. If $\gamma$ is a strictly singular extremal, the Prontryagin Maximum Principle yields a non-zero dual curve $\lambda$ satisfying along $\gamma$ the additional equations

$$
0=\left\langle\lambda, f_{1}\right\rangle=\lambda_{1} \quad \text { and } \quad 0=\left\langle\lambda, f_{2}\right\rangle=\lambda_{2}\left(1-\gamma_{1}\right)+\lambda_{3} \gamma_{1}^{p} \quad \text { on }[0,1] .
$$


The Goh condition $\left\langle\lambda,\left[f_{1}, f_{2}\right]\right\rangle=0$ along $\gamma$ implies the further relation $\lambda_{2}=p \lambda_{3} \gamma_{1}^{p-1}$. Now, using $\left|\gamma_{1}\right| \leq 1$, it is not difficult to see that $\lambda_{2}=0$ and thus $\gamma_{1}=0$. Then we have $u_{1}=0$ and thus $\left|u_{2}\right|=1$. In particular $\gamma(t)=(0, t, 0)$ is a singular extremal, whose dual curve is constant $\lambda(t)=(0,0,1)$, and whose control, $u(t)=(0,1)$, is constant as well.

We claim that the curve $\gamma(t)=(0, t, 0)$ is strictly singular. Indeed, any regular extremal $\gamma$ together with its dual curve $\lambda$ is a characteristic curve of the following Hamiltonian system. Let $H: T^{*} \mathbb{R}^{3}=\mathbb{R}^{3} \times \mathbb{R}^{3} \rightarrow \mathbb{R}$ be the Hamiltonian

$$
H(x, \lambda)=\frac{1}{2}\left(\left\langle\lambda, f_{1}(x)\right\rangle^{2}+\left\langle\lambda, f_{2}(x)\right\rangle^{2}\right) .
$$

If $\gamma$ is regular, then the pair $(\gamma, \lambda)$ solves the system of ordinary differential equations $\dot{\gamma}=$ $H_{\lambda}(\gamma, \lambda)$ and $\dot{\lambda}=-H_{x}(\gamma, \lambda)$. In particular, $\gamma$ is smooth.

Now assume by contradiction that for the curve $\gamma(t)=(0, t, 0)$ there exists an absolutely continuous curve of covectors $\lambda$ such that $\dot{\gamma}=H_{\lambda}(\gamma, \lambda)$ and $\dot{\lambda}=-H_{x}(\gamma, \lambda)$. If $\gamma$ satisfies the first equation, it follows that $\lambda_{1}=0$ and $\lambda_{2}=1$. From the second equation it then follows that $\dot{\lambda}_{1}=\lambda_{2}^{2}=1$, that is a contradiction.

When $p>2$, we have $\left[f_{1},\left[f_{1}, f_{2}\right]\right](\gamma(t))=\left[f_{2},\left[f_{1}, f_{2}\right]\right](\gamma(t))=0$, and thus $\gamma$ is not even "regular abnormal" in the sense of [24, Section 6]. Now we show that when $p$ is an even integer the singular curve $\gamma$ is locally length-minimizing.

Proof of Theorem 1.3 - (ii). The precise claim we prove is the following: let $p$ be an even integer and $t_{0}:=\frac{2}{p+1}$. For any compact interval $[a, b] \subset \mathbb{R}$ such that $b-a<t_{0}$, the segment $\gamma(t)=(0, t, 0), t \in[a, b]$, is the unique length-minimizing curve in $\left(\mathbb{R}^{3}, \Delta, g\right)$ joining the point $(0, a, 0)$ to $(0, b, 0)$. The proof follows an idea of [24, Section 7.1].

Let $\tau:=b-a<t_{0}$ and let $\eta:[0, \tau] \rightarrow \mathbb{R}^{3}$ be any horizontal curve parameterized by arclength such that $\eta(0)=(0, a, 0)$ and $\eta(\tau)=(0, b, 0)$. Then there exist measurable functions $u_{1}, u_{2}$ (unique up to sets of measure zero) such that

$$
\dot{\eta}_{1}=u_{1}, \quad \dot{\eta}_{2}=u_{2}\left(1-\eta_{1}\right), \quad \dot{\eta}_{3}=u_{2} \eta_{1}^{p} \text { a.e. on }[0, \tau] .
$$

We claim that, under the hypothesis that $\int_{0}^{\tau} u_{1} d t=\int_{0}^{\tau} u_{2} \eta_{1}^{p} d t=0$, one has

$$
\int_{0}^{\tau} u_{2}\left(1-\eta_{1}\right) d t \leq \tau
$$

with equality holding only for $u_{1}=0$ and $u_{2}=1$ a.e. on $[0, \tau]$.

Using this claim, the proof can be concluded in the following way. If $\gamma^{\prime}$ is another admissible curve joining $(0, a, 0)$ and $(0, b, 0)$ in time $\tau-\varepsilon<\tau$, we construct another curve $\gamma^{\prime \prime}$ by attaching to $\gamma^{\prime}$ a straight segment back and forth from $(0, b, 0)$, for a total time $\varepsilon$. But then, the inequality in (5.1) would be strict, and we have an absurd. Moreover, we observe that length $(\gamma)=\tau=$ $\eta_{2}(\tau)-\eta_{2}(0)=\int_{0}^{\tau} u_{2}\left(1-\eta_{1}\right) d t$. So we have equality in (5.1), and from the claim it follows that $\eta=\gamma$. This proves that $\gamma$ is the unique length-minimizing curve between its end-points. Since

We prove (5.1). Let us define the function $V(s)=\int_{0}^{s} u_{2} d t$ for $s \in[0, \tau]$ and $\beta:=\left\|\eta_{1}\right\|_{L^{\infty}([0, \tau] ; \mathbb{R})}$.

$$
\int_{0}^{\tau} u_{2}\left(1-\eta_{1}\right) d t \leq V(\tau)+\tau \beta=\tau-(\tau-V(\tau))+\beta \tau,
$$

it suffices to show that $\beta \leq \frac{\tau-V(\tau)}{\tau}$, that is a consequence of the following inequalities:

$$
t_{0} \beta^{p+1} \leq \int_{0}^{\tau} \eta_{1}^{p} d t \leq \beta^{p}(\tau-V(\tau)) .
$$


The inequality in the right-hand side follows easily, since

$$
\int_{0}^{\tau} \eta_{1}^{p} d t=\int_{0}^{\tau} \eta_{1}^{p}\left(1-u_{2}\right) d t
$$

To prove the inequality in the left-hand side of (5.2), we fix $t_{\max } \in[0, \tau]$ such that $\left|\eta_{1}\left(t_{\max }\right)\right|=\beta$. Since $\eta_{1}(0)=\eta_{1}(\tau)=0$ and $\left|\dot{\eta}_{1}\right| \leq 1$ a.e. on $[0, \tau]$, we have $\min \left\{t_{\max }, \tau-t_{\max }\right\} \geq \beta$, meaning that the intervals $I_{1}=\left[t_{\max }-\beta, t_{\max }\right]$ and $I_{2}=\left[t_{\max }, t_{\max }+\beta\right]$ are contained in $[0, \tau]$. These arguments also imply that on $I_{1}$ and $I_{2},\left|\eta_{1}\right|$ is bounded from below by linear functions $\ell_{1}$ and $\ell_{2}$, respectively, such that $\ell_{1}\left(t_{\max }-\beta\right)=\ell_{2}\left(t_{\max }+\beta\right)=0$ and $\ell_{1}\left(t_{\max }\right)=\ell_{2}\left(t_{\max }\right)=\beta$. Since the exponent $p$ is even, this implies that $\int_{0}^{\tau} \eta_{1}^{p} d t \geq \frac{2}{p+1} \beta^{p+1}$, as desired.

Clearly, if $\left(u_{1}, u_{2}\right)=(0,1)$ a.e. on $[0, \tau]$ we have equality in (5.1). Conversely, assuming that equality holds we deduce that $\beta=(\tau-V(\tau)) \frac{p+1}{2}$, whence from (5.2) we obtain

$$
\int_{0}^{\tau} \eta_{1}^{p}\left(1-u_{2}\right) d t=\beta^{p}(\tau-V(\tau))
$$

which holds if and only if $\eta_{1}=\beta$ on $[0, \tau]$. Since $\eta_{1}(0)=0$, then $\eta_{1}=0$, and this concludes the proof.

The first case where the previous argument fails is when $p=3$. In this case, to the best of our knowledge it is an open question to decide whether the curve $\gamma(t)=(0, t, 0)$ is minimizing or not. Using Theorem 1.1, we will see that the answer is in the negative.

Proof of Theorem 1.3 - (iii). Let $F=F_{q_{0}}: L^{1}\left([0,1] ; \mathbb{R}^{2}\right) \rightarrow \mathbb{R}^{3}$ be the end-point map with initial point $q_{0}=0$ introduced in (3.8). We claim that $F$ is open at the point $u=(0,1) \in$ $L^{1}\left([0,1] ; \mathbb{R}^{2}\right)$, the control of the singular trajectory $\gamma$. As in $(3.9)$ and $(3.11)$, we let $G(v)=$ $G_{q_{1}}(v)=F_{q_{0}}(u+v)$, where $q_{1}=(0,1,0)$. The infinitesimal analysis of $F$ at $u$ is reduced to the infinitesimal analysis of $G$ at 0 . By Lemma 3.3 the differential of $G$ at 0 is given by $d_{0} G(v)=\int_{0}^{1} g_{v(t)}^{t} d t$, where

$$
g_{v(t)}^{t}=\sum_{i=1}^{2} v_{i}(t) \operatorname{Ad}\left(\overrightarrow{\exp } \int_{1}^{t} f_{u(\tau)} d \tau\right) f_{i},
$$

and $\operatorname{Ad}\left(\overrightarrow{\exp } \int_{1}^{t} f_{u(\tau)} d \tau\right)$ is the differential of the inverse of the flow $(x, t) \mapsto P_{1}^{t}(x)$, where $x=$ $\left(x_{1}, x_{2}, x_{3}\right) \in \mathbb{R}^{3}, t \in[0,1]$, and $P_{1}^{t}(x)=\gamma(t)$, where $t \mapsto \gamma(t)$ is the horizontal trajectory with control $u$ such that $\gamma(1)=x \in \mathbb{R}^{3}$. Using the formulas for $f_{1}$ and $f_{2}$ in (1.4), we find

$$
\gamma_{1}(t)=x_{1}, \quad \gamma_{2}(t)=(t-1)\left(1-x_{1}\right)+x_{2}, \quad \gamma_{3}(t)=(t-1) x_{1}^{3}+x_{3} .
$$

The inverse of the differential $\left(P_{1}^{t}\right)_{*}^{-1}=\left(P_{t}^{1}\right)_{*}: T_{(0, t, 0)} \mathbb{R}^{3} \rightarrow T_{(0,1,0)} \mathbb{R}^{3}$ is given by

$$
\left(P_{t}^{1}\right)_{*}=\left(\begin{array}{ccc}
1 & 0 & 0 \\
t-1 & 1 & 0 \\
-3(t-1) x_{1}^{2} & 0 & 1
\end{array}\right) .
$$

Accordingly, the vector fields $g_{1}^{t}$ and $g_{2}^{t}$ are

$$
\begin{aligned}
g_{1}^{t} & :=\left(P_{t}^{1}\right)_{*} f_{1}=\frac{\partial}{\partial x_{1}}+(t-1) \frac{\partial}{\partial x_{2}}-3(t-1) x_{1}^{2} \frac{\partial}{\partial x_{3}}, \\
g_{2}^{t} & :=\left(P_{t}^{1}\right)_{*} f_{2}=f_{2},
\end{aligned}
$$


and we obtain the following formula for the differential of $G$ :

$$
d_{0} G(v)=\left(\begin{array}{c}
\int_{0}^{1} v_{1}(t) d t \\
\int_{0}^{1}\left\{(t-1) v_{1}(t)+v_{2}(t)\right\} d t \\
0
\end{array}\right) .
$$

We then see that a generator of $\operatorname{Im}\left(d_{0} G\right)^{\perp}$ is the covector $\lambda=(0,0,1)$.

We compute the intrinsic Hessian $\mathcal{D}_{0}^{2} G$, again using Lemma 3.3. By (5.3), for every $0 \leq$ $t_{1}, t_{2} \leq 1$ and every $v, w \in \mathbb{R}^{2}$ at the point $q_{1}=(0,1,0)$ we have $\left\langle\lambda,\left[g_{v}^{t_{1}}, g_{w}^{t_{2}}\right]\left(q_{1}\right)\right\rangle=0$. Then $\lambda d_{0}^{2} G(v)=0$ for every $v \in \operatorname{ker}\left(d_{0} G\right)$, hence $\mathcal{D}_{0}^{2} G=0$.

Finally, we compute the intrinsic third differential $\mathcal{D}_{0}^{3} G$. Note first that since the intrinsic Hessian vanishes, by our definition in (2.3) we also have $\operatorname{dom}\left(\mathcal{D}_{0}^{3} G\right)=\operatorname{ker}\left(d_{0} G\right)$. The only commutator of length three which has non-zero third component is $\left[g_{1}^{t_{1}},\left[g_{1}^{t_{2}}, g_{1}^{t_{3}}\right]\right]\left(q_{1}\right)$, and namely we have

$$
\left\langle\lambda,\left[g_{1}^{t_{1}},\left[g_{1}^{t_{2}}, g_{1}^{t_{3}}\right]\right]\left(q_{1}\right)\right\rangle=6\left(t_{2}-t_{3}\right) .
$$

Then, again by Lemma 3.3, for $v=(2 \pi \sin (2 \pi t), 1) \in \operatorname{ker}\left(d_{0} G\right)=\operatorname{dom}\left(\mathcal{D}_{0}^{3} G\right)$, the third differential is

$$
\lambda \mathcal{D}_{0}^{3} G(v)=2 \int_{0}^{1} \int_{0}^{t_{1}} \int_{0}^{t_{2}} 6 v_{1}\left(t_{1}\right) v_{1}\left(t_{2}\right) v_{1}\left(t_{3}\right)\left(t_{2}-t_{3}\right) d t_{3} d t_{2} d t_{1}=15 \neq 0 .
$$

Hence, by Theorem 1.1 the mapping $G$ is open at 0 and thus the singular trajectory $\gamma$ is not optimal (i.e., of minimal length). Alternatively, we could have used Theorem 1.2 to deduce that $\lambda=0$, contradicting Pontryagin Maximum Principle.

\section{REFERENCES}

[1] A. A. Agrachev. Some open problems. In Geometric control theory and sub-Riemannian geometry, volume 5 of Springer INdAM Ser., pages 1-13. Springer, Cham, 2014

[2] A. A. Agrachev, F. Boarotto, and A. Lerario. Homotopically invisible singular curves. Calc. Var. Partial Differential Equations, 56(4):Art. 105, 34, 2017.

[3] A. A. Agrachev and R. V. Gamkrelidze. The shuffle product and symmetric groups. In Differential equations, dynamical systems, and control science, volume 152 of Lecture Notes in Pure and Appl. Math., pages 365382. Dekker, New York, 1994.

[4] A. A. Agrachev, A. Gentile, and A. Lerario. Geodesics and horizontal-path spaces in Carnot groups. Geom. Topol., 19(3):1569-1630, 2015.

[5] A. A. Agrachev and Y. L. Sachkov. Control theory from the geometric viewpoint, volume 87 of Encyclopaedia of Mathematical Sciences. Springer-Verlag, Berlin, 2004. Control Theory and Optimization, II.

[6] A. A. Agrachev and A. V. Sarychev. Abnormal sub-Riemannian geodesics: Morse index and rigidity. Ann. Inst. H. Poincaré Anal. Non Linéaire, 13(6):635-690, 1996.

[7] A. V. Arutyunov and D. Y. Karamzin. Regular zeros of quadratic mappings and their applications. Mat. $S b ., 202(6): 3-28,2011$.

[8] D. Barilari and F. Boarotto. On the set of points of smoothness for the value function of affine optimal control problems. SIAM J. Control Optim., 56(2):649-671, 2018.

[9] D. Barilari, Y. Chitour, F. Jean, D. Prandi, and M. Sigalotti. On the regularity of abnormal minimizers for rank 2 sub-riemannian structures. J. Math. Pures Appl., 2019.

[10] A. Belotto da Silva and L. Rifford. The Sard conjecture on Martinet surfaces. Duke Math. J., 167(8):14331471,2018

[11] F. Boarotto and A. Lerario. Homotopy properties of horizontal path spaces and a theorem of Serre in subriemannian geometry. Comm. Anal. Geom., 25(2):269-301, 2017.

[12] F. Boarotto and M. Sigalotti. Time-optimal trajectories of generic control-affine systems have at worst iterated Fuller singularities. Ann. Inst. H. Poincaré Anal. Non Linéaire, 36(2):327-346, 2019.

[13] B. Bonnard and I. Kupka. Generic properties of singular trajectories. Ann. Inst. H. Poincaré Anal. Non Linéaire, 14(2):167-186, 1997. 
[14] Y. Chitour, F. Jean, and E. Trélat. Genericity results for singular curves. J. Differential Geom., 73(1):45-73, 2006.

[15] Y. Chitour, F. Jean, and E. Trélat. Singular trajectories of control-affine systems. SIAM J. Control Optim., 47(2):1078-1095, 2008.

[16] E. A. Coddington and N. Levinson. Theory of ordinary differential equations. McGraw-Hill Book Company, Inc., New York-Toronto-London, 1955.

[17] B. S. Goh. Necessary conditions for singular extremals involving multiple control variables. SIAM J. Control, 4:716-731, 1966.

[18] E. Hakavuori and E. Le Donne. Non-minimality of corners in subriemannian geometry. Invent. Math., 206(3):693-704, 2016.

[19] I. Kupka. Counterexample to the Morse-Sard theorem in the case of infinite-dimensional manifolds. Proc. Amer. Math. Soc., 16:954-957, 1965.

[20] E. Le Donne, G. P. Leonardi, R. Monti, and D. Vittone. Extremal curves in nilpotent Lie groups. Geom. Funct. Anal., 23(4):1371-1401, 2013.

[21] E. Le Donne, G. P. Leonardi, R. Monti, and D. Vittone. Extremal polynomials in stratified groups. Comm. Anal. Geom., 26(4):723-757, 2018.

[22] E. Le Donne, R. Montgomery, A. Ottazzi, P. Pansu, and D. Vittone. Sard property for the endpoint map on some Carnot groups. Ann. Inst. H. Poincaré Anal. Non Linéaire, 33(6):1639-1666, 2016.

[23] G. P. Leonardi and R. Monti. End-point equations and regularity of sub-Riemannian geodesics. Geom. Funct. Anal., 18(2):552-582, 2008.

[24] W. Liu and H. J. Sussmann. Shortest paths for sub-Riemannian metrics on rank-two distributions. Mem. Amer. Math. Soc., 118(564):x+104, 1995.

[25] R. Montgomery. Abnormal minimizers. SIAM J. Control Optim., 32(6):1605-1620, 1994.

[26] R. Montgomery. A tour of subriemannian geometries, their geodesics and applications, volume 91 of Mathematical Surveys and Monographs. American Mathematical Society, Providence, RI, 2002.

[27] R. Monti. The regularity problem for sub-Riemannian geodesics. In Geometric control theory and subRiemannian geometry, volume 5 of Springer INdAM Ser., pages 313-332. Springer, Cham, 2014.

[28] R. Monti. Regularity results for sub-Riemannian geodesics. Calc. Var. Partial Differential Equations, 49(12):549-582, 2014

[29] R. Monti. The regularity problem for geodesics of the control distance. BPMAS, pages 137-146, 2018.

[30] R. Monti, A. Pigati, and D. Vittone. Existence of tangent lines to Carnot-Carathéodory geodesics. Calc. Var. Partial Differential Equations, 57(3):Art. 75, 18, 2018.

[31] L. S. Pontryagin, V. G. Boltyanskii, R. V. Gamkrelidze, and E. F. Mishchenko. The mathematical theory of optimal processes. Translated by D. E. Brown. A Pergamon Press Book. The Macmillan Co., New York, 1964.

[32] L. Rifford and E. Trélat. Morse-Sard type results in sub-Riemannian geometry. Math. Ann., 332(1):145-159, 2005.

[33] I. R. Shafarevich. Basic algebraic geometry. Springer-Verlag, New York-Heidelberg, 1974. Translated from the Russian by K. A. Hirsch, Die Grundlehren der mathematischen Wissenschaften, Band 213.

[34] Y. Shitov. A counterexample to Comon's conjecture. SIAM J. Appl. Algebra Geom., 2(3):428-443, 2018.

[35] H. J. Sussmann. High-order open mapping theorems. In Directions in mathematical systems theory and optimization, volume 286 of Lect. Notes Control Inf. Sci., pages 293-316. Springer, Berlin, 2003.

Dipartimento di Matematica Tullio Levi-Civita, Università di Padova, Italy

E-mail address: francesco.boarotto@math.unipd.it

Dipartimento di Matematica Tullio Levi-Civita, Università di Padova, Italy

E-mail address: monti@math.unipd.it

ETH Zürich Department Mathematik, RÄmistrasse 101, CH-8093 Zürich, Switzerland

E-mail address: francesco.palmurella@math.ethz.ch 